\title{
Confidence interval for correlation estimator between latent processes
}

\author{
Akitoshi Kimura * \\ August 21, 2018
}

\begin{abstract}
Kimura and Yoshida 26 treated a model in which the finite variation part of a two-dimensional semimartingale is expressed by time-integration of latent processes. They proposed a correlation estimator between the latent processes and proved its consistency and asymptotic mixed normality. In this paper, we discuss the confidence interval of the correlation estimator to detect the correlation. We propose two types of estimators for asymptotic variance of the correlation estimator and prove their consistency in a high frequency setting. Our model includes doubly stochastic Poisson processes whose intensity processes are correlated Itô processes. We compare our estimators based on the simulation of the doubly stochastic Poisson processes.
\end{abstract}

\section{Introduction}

Integrated correlation is an important index in high frequency financial data analysis. Epps [16] pointed out that the sample correlation between the returns of two different stocks decreases as the sampling frequency of data increases. It is considered that non-synchronicity and market microstructure of trading cause this phenomenon.

Non-synchronous covariance estimation schemes have been developed: Fourier analytic approach (Malliavin and Mancino [32, Malliavin et al. [33]) and the cumulative covariance estimator (Hayashi and Yoshida 23, 21, 22, Mykland 40]).

The market microstructure is modeled as the noise added to the latent price process. This modeling was successful and denoising techniques have also been developed: sub-sampling (Zhang et al. [48, Zhang [49]), pre-averaging (Podolskij and Vetter [43, Jacod et al. [25]), and others (Zhou [50]). There are many studies that treat both non-synchoronicity and market microstructure noise: Malliavin and Mancino 34, Mancino and Sanfelici 35, Park and Linton 42, Voev and Lunde [47, Griffin and Oomen [19, Christensen et al. [12, 13, Koike [27, 29, 28, Aït-Sahalia et al. 4], Barndorff-Nielsen et al. [6], Bibinger [8, 9].

The followings are proposed as the cause of market microstructure: bid-ask spread (Roll [4]), discretization error (Gottlieb and Kalay [18), and asymmetric information (Glosten and Milgrom [17]). On the other hand, the relationship

\footnotetext{
*Graduate School of Mathematical Sciences, University of Tokyo, 3-8-1 Komaba, Meguroku, Tokyo 153-8914, Japan, Japan Science and Technology Agency, CREST
} 
between the additive noise modeling and the market microstructure is not so clearly explained.

In ultra high frequency sampling, recently, the market microstructure is modeled as dynamics of the limit order book (LOB) rather than the noise. Many studies adopt approaches modeling LOB with Poisson processes (Cont et al. [15], Abergel and Jedidi [2, Muni Toke [46, 37, Smith et al. 45, Muni Toke and Yoshida 39]), Hawkes processes (Hewlett [24, Large [31, Bowsher 10, Bacry et al. 5, Abergel and Jedidi 3, Muni Toke and Pomponio 38, Muni Toke [36], Clinet and Yoshida [14, Ogihara and Yoshida [1]), and doubly stochastic Poisson processes (Abergel et al. 1], Guilbaud and Pham [20, Chertok et al. [1], Korolev et al. [30]). These approaches can also treat the non-synchronicity of trading.

In this stream, Kimura and Yoshida 26 focused on the integrated correlation between intensity processes of doubly stochastic Poisson processes. They introduced the following model to treat it in a generalized form. This paper is also based on the model.

Now, we consider a stochastic basis $\mathcal{B}=(\Omega, \mathcal{F}, \mathbb{F}, P), \mathbb{F}=\left(\mathcal{F}_{t}\right)_{t \in[0, T]}$. On $\mathcal{B}$, let $\mathbb{X}=\left(X^{1}, X^{2}\right)$ be an $\mathbb{R}^{2}$-valued Itô process given by

$$
\mathbb{X}_{t}=\mathbb{X}_{0}+\int_{0}^{t} \mathbb{X}_{s}^{0} d s+\int_{0}^{t} \mathbb{X}_{s}^{1} d w_{s} \quad(t \in[0, T]),
$$

where $w$ is an $r$-dimensional $\mathcal{F}$-Wiener process, $\mathbb{X}_{0}$ is an $\mathbb{F}_{0}$-measurable random variable, $\mathbb{X}^{0}$ is a two-dimensional $\mathbb{F}$-adapted process, and $\mathbb{X}^{1}$ is an $\mathbb{R}^{2} \otimes \mathbb{R}^{r}$ valued $\mathbb{F}$-adapted process satisfies condition $[\mathrm{A}]$ mentioned later. Let $a_{n}$ be a positive number depending on $n$. On $\mathcal{B}$, consider a two-dimensional measurable process $\mathbb{Y}^{n}=\left(Y^{n, 1}, Y^{n, 2}\right)$ having a decomposition

$$
\mathbb{Y}_{t}^{n}=\mathbb{Y}_{0}^{n}+\int_{0}^{t} a_{n} \mathbb{X}_{s} d s+\mathbb{M}_{t}^{n} \quad(t \in[0, T]),
$$

where $\mathbb{M}^{n}$ is a two-dimensional measurable process with $\mathbb{M}_{0}^{n}=0$ satisfies condition $[\mathrm{B}],\left[\mathrm{B}^{\prime}\right]$, or $\left[\mathrm{B}^{\sharp \sharp}\right]$ mentioned later.

In this model, $\mathbb{Y}$ can be a counting process with intensity process $a_{n} \mathbb{X}$ where $\mathbb{X}$ is $\mathbb{R}_{+}^{2}$-valued, i.e. $\mathbb{Y}$ is a two-dimensional doubly stochastic Poisson process. This counting process models the high frequency counting data of the orders or transactions in the active market, for example.

Let $I_{j}=\left[t_{j-1}, t_{j}\right)$ for a sampling design $\Pi=\left(t_{j}\right)_{j=0, \ldots, b_{n}}$ with $0=t_{0}<t_{1}<$ $\cdots<t_{b_{n}}=T$ and $h_{j}=t_{j}-t_{j-1}$.

Kimura and Yoshida [26] introduced the estimator of the covariance $\left\langle X^{1}, X^{2}\right\rangle$ from the sampled data of $\mathbb{Y}$ as follows. For $\alpha, \beta=1,2$,

$$
S_{n}^{\alpha \beta}=\sum_{k=2}^{b_{n}}\left(\frac{Y_{t_{k}}^{n, \alpha}-Y_{t_{k-1}}^{n, \alpha}}{a_{n} h_{k}}-\frac{Y_{t_{k-1}}^{n, \alpha}-Y_{t_{k-2}}^{n, \alpha}}{a_{n} h_{k-1}}\right)\left(\frac{Y_{t_{k}}^{n, \beta}-Y_{t_{k-1}}^{n, \beta}}{a_{n} h_{k}}-\frac{Y_{t_{k-1}}^{n, \beta}-Y_{t_{k-2}}^{n, \beta}}{a_{n} h_{k-1}}\right),
$$

Here, $S_{n}=\left(S_{n}^{12}, S_{n}^{11}, S_{n}^{22}\right)^{\star}$ is the (co)variance estimator. $S_{n}$ depends on the scaling parameter $a_{n}$ which is not derived from the data of $\mathbb{Y}$. Therefore, treat the correlation estimator $C_{n}^{12}=S_{n}^{12} / \sqrt{S_{n}^{11} S_{n}^{22}}$ which does not depend on the scaling parameter $a_{n}$. 
Let

$$
U^{\alpha \beta}=\frac{2}{3}\left\langle X^{\alpha, c}, X^{\beta, c}\right\rangle_{T}=\frac{2}{3} \int_{0}^{T} X_{t}^{\alpha 1} \cdot X_{t}^{\beta 1} d t \quad(\alpha, \beta=1,2)
$$

and $U=\left(U^{12}, U^{11}, U^{22}\right)^{\star}$, where $X^{\alpha, c}$ is the continuous part of $X^{\alpha}$ and $X_{t}^{\alpha 1}$ is the $\alpha$-th row of $\mathbb{X}_{t}^{1}$. Let

$$
\begin{aligned}
\gamma^{\left(\alpha_{1}, \beta_{1}\right),\left(\alpha_{2}, \beta_{2}\right)} & =\int_{0}^{T} \sum_{i, j=1}^{r} \frac{X_{i, s}^{\alpha_{1} 1} X_{j, s}^{\beta_{1} 1}+X_{j, s}^{\alpha_{1} 1} X_{i, s}^{\beta_{1} 1}}{2} \frac{X_{i, s}^{\alpha_{2} 1} X_{j, s}^{\beta_{2} 1}+X_{j, s}^{\alpha_{2} 1} X_{i, s}^{\beta_{2} 1}}{2} d s \\
& =\int_{0}^{T}\left(\mathbb{X}_{s}^{1}\right)^{\tilde{\otimes}\left(\alpha_{1}, \beta_{1}\right)} \cdot\left(\mathbb{X}_{s}^{1}\right)^{\tilde{\otimes}\left(\alpha_{2}, \beta_{2}\right)} d s
\end{aligned}
$$

and $\Gamma=\left(\gamma^{p q}\right)_{p, q=(1,2),(1,1),(2,2)}$, where $X_{i, s}^{\alpha 1}$ is the $(\alpha, i)$-element of $\mathbb{X}_{s}^{1}$.

Under $[\mathrm{A}]$ and any one of $[\mathrm{B}],\left[\mathrm{B}^{\prime}\right]$, and $\left[\mathrm{B}^{\sharp}\right]$, Kimura and Yoshida [26 proved the the asymptotic mixed normality of the covariance estimator and the correlation estimator.

$$
\left(\frac{T}{b_{n}}\right)^{-1 / 2}\left(S_{n}-U\right) \rightarrow^{d_{s}} \Gamma^{1 / 2} \zeta
$$

as $n \rightarrow \infty$, where $\zeta$ is an $\mathbb{R}^{3}$-valued standard normal variable independent of $\mathcal{F}$. and $d_{s}$ means $\mathcal{F}$-stable convergence. Let $R=U^{12} / \sqrt{U^{11} U^{22}}$ and suppose that $U^{11} U^{22} \neq 0$ a.s.

$$
\left(\frac{T}{b_{n}}\right)^{-1 / 2}\left(C_{n}^{12}-R\right) \rightarrow^{d_{s}} \Xi^{1 / 2} \zeta
$$

as $n \rightarrow \infty$, where

$$
\Xi^{1 / 2}:=\left(\frac{1}{\sqrt{U^{11} U^{22}}}, \frac{-U^{12}}{2 \sqrt{\left(U^{11}\right)^{3} U^{22}}}, \frac{-U^{12}}{2 \sqrt{U^{11}\left(U^{22}\right)^{3}}}\right) \Gamma^{1 / 2} .
$$

$\Xi=\left(\Xi^{1 / 2}\right)\left(\Xi^{1 / 2}\right)^{\star}$ is the asymptotic variance of the correlation estimator.

In this paper, we introduce two types of estimators for $\Gamma$ and prove their consistency in order to obtain the consistent estimator for $\Xi$. Therefore, we can detect the correlation between latent processes based on hypothesis testing.

This paper is organized as follows. In Section 2 we list the assumptions. In Section 3. we introduce the estimators for $\Gamma$ and $\Xi$, and state the main result: their consistency. In Section 4 the results in Section 3 are proved. In Section 5. an example and simulation studies are given.

\section{Assumptions}

For simplicity, we assume $t_{j}=j\left(T / b_{n}\right), h_{j}=T / b_{n}=: \delta_{n}$. We write $\Delta_{j} V=$ $V_{t_{j}}-V_{t_{j-1}}$ for a process $V$. Consider the following conditions.

[A] Process $\mathbb{X}$ admits the representation (1.1) for an $\mathbb{R}^{2}$-valued $\mathcal{F}_{0}$-measurable random variable $\mathbb{X}_{0}$ and coefficients $\mathbb{X}^{\kappa}(\kappa=0,1)$ such that $\mathbb{X}^{0}$ is a cádlág $\mathbb{F}$-adapted process and that $\mathbb{X}^{1}$ has a representation

$$
\mathbb{X}_{t}^{1}=\mathbb{X}_{0}^{1}+\int_{0}^{t} \mathbb{X}_{s}^{10} d s+\int_{0}^{t} \sum_{\kappa^{\prime}=1}^{r^{\prime}} \mathbb{X}_{s}^{1 \kappa^{\prime}} d \tilde{w}_{s}^{\kappa^{\prime}} \quad(t \in[0, T]),
$$


where $\mathbb{X}_{0}^{1}$ is an $\mathbb{R}^{2} \otimes \mathbb{R}^{r}$-valued $\mathcal{F}_{0}$-measurable random variable, $\tilde{w}=$ $\left(\tilde{w}^{1}, \ldots, \tilde{w}^{r^{\prime}}\right)$ is an $r^{\prime}$-dimensional $\mathbb{F}$-Wiener process (not necessary independent of $w)$, and $\mathbb{X}_{s}^{1 \kappa^{\prime}}\left(\kappa^{\prime}=0,1, \ldots, r^{\prime}\right)$ are $\mathbb{R}^{2} \otimes \mathbb{R}^{r}$-valued cádlág $\mathbb{F}$-adapted processes.

[B] $\mathbb{M}^{n}=\left(M^{n, \alpha}\right)_{\alpha=1,2}$ is a two-dimensional $\mathbb{F}$-local martingale with $\mathbb{M}_{0}^{n}=0$ and such that

(i) $\lim _{n \rightarrow \infty} b_{n}^{5 / 2} / a_{n}=0$.

(ii) $\sum_{j=1}^{b_{n}}\left|\Delta_{j} \mathbb{M}^{n}\right|^{2}=O_{p}\left(a_{n}\right)$ as $n \rightarrow \infty, \sup _{t \in[0,1]}\left|\Delta \mathbb{M}^{n}\right| \leq c a_{n}^{1 / 2}$ for a constant $\mathrm{c}$ independent of $\mathrm{n}$.

(iii) The absolutely continuous (w.r.t. the Lebesgue measure a.s.) mapping $[0, T] \ni t \mapsto\left\langle\mathbb{M}^{n}, w\right\rangle_{t} \in \mathbb{R}^{2} \otimes \mathbb{R}^{r}$ satisfies $\sup _{t \in[0, T]}\left|d\left\langle\mathbb{M}^{n}, w\right\rangle_{t} / d t\right|=$ $O_{p}\left(b_{n}\right)$ as $n \rightarrow \infty$.

Here, $\left\langle\mathbb{M}^{n}, w\right\rangle_{t}$ is the $2 \times r$ matrix of angle brackets $\left\langle M^{n, \alpha}, w^{k}\right\rangle_{t}$ for $\mathbb{M}^{n}=$ $\left(M^{n, \alpha}\right)_{\alpha=1,2}$ and $w=\left(w^{k}\right)_{k=1, \ldots, r}$.

[B'] $\mathbb{M}^{n}$ is a two-dimensional $\mathbb{F}$-local martingale with $\mathbb{M}_{0}^{n}=0$, satisfies [B] (i), (iii), and

(ii') $E\left[\sum_{j=1}^{b_{n}}\left|\Delta_{j} \mathbb{M}^{n}\right|^{2}\right]=O\left(a_{n}\right)$ as $n \rightarrow \infty$.

$\left[\mathbf{B}^{\sharp}\right] \quad \mathbb{M}^{n}$ is a two-dimensional $\mathbb{F}$-local martingale with $\mathbb{M}_{0}^{n}=0$, satisfies [B] (ii), (iii), and

$\left(\mathrm{i}^{\sharp}\right) \lim _{n \rightarrow \infty} b_{n}^{3} / a_{n}=0$.

[C] $E\left[\sum_{j=1}^{b_{n}}\left|\Delta_{j} \mathbb{M}^{n}\right|^{4}\right]=O\left(a_{n}^{2} b_{n}^{-1}\right)$ as $n \rightarrow \infty$

Remark 2.1. $[A],[B]$, and $\left[B^{\prime}\right]$ are the same condition in [26]. [B $\left[B^{\sharp}\right]$ is a bit stronger condition than $\left[B^{\sharp}\right]$ in [26]].

Remark 2.2. The doubly stochastic Poisson process model satisfies [C].

\section{Results}

We need the estimator of $\Gamma$ to obtain the asymptotic variance estimator. Here, we give two types of the gamma estimator.

Before that, we introduce some notations to simplify the description. Let $A^{\otimes(i, j)}$ is an $(i, j)$-element of $A^{\otimes}, A^{\otimes}=A \otimes A=A A^{\star}$, and ${ }^{\star}$ denotes transpose. Let $x \tilde{\otimes} y=\left(\left(x_{i} y_{j}+x_{j} y_{i}\right) / 2\right) \in \mathbb{R}^{r} \otimes \mathbb{R}^{r}$ for $x=\left(x_{i}\right), y=\left(y_{i}\right) \in \mathbb{R}^{r}$, and let $x^{\tilde{\otimes}(\alpha, \beta)}=x^{\alpha} \tilde{\otimes} x^{\beta}$. for $x=\left(x_{i}^{\alpha}\right) \in \mathbb{R}^{2} \otimes \mathbb{R}^{r}$. We write $x \cdot y=\sum_{i=1}^{r} x_{i} y_{i}$ for $x=\left(x_{i}\right)$, $y=\left(y_{i}\right) \in \mathbb{R}^{r}$, and $x \cdot y=\sum_{i, j=1}^{r} x_{i, j} y_{i, j}$ for $x=\left(x_{i, j}\right), y=\left(y_{i, j}\right) \in \mathbb{R}^{r} \otimes \mathbb{R}^{r}$. Write

$$
\tilde{V}_{k}:=\frac{\Delta_{k} V}{a_{n} h_{k}}=\frac{\Delta_{k} V}{a_{n} \delta_{n}} .
$$

for a stochastic process $V$. For example, we can write

$$
S_{n}^{\alpha \beta}=\sum_{j=2}^{b_{n}}\left(\tilde{\mathbb{Y}}_{j}^{n}-\tilde{\mathbb{Y}}_{j-1}^{n}\right)^{\otimes(\alpha, \beta)}, \quad \gamma^{p q}=\int_{0}^{1}\left(\mathbb{X}_{s}^{1}\right)^{\tilde{\otimes} p} \cdot\left(\mathbb{X}_{s}^{1}\right)^{\tilde{\otimes} q} d s .
$$




\section{1 $\Gamma$ estimator}

In the similar way in Barndorff-Nielsen and Shephard [7, we define

$$
\begin{aligned}
\hat{\gamma}_{n, 1}^{p q}=\frac{9}{8}\{ & \sum_{k=2}^{b_{n}}\left(\tilde{\mathbb{Y}}_{k}^{n}-\tilde{\mathbb{Y}}_{k-1}^{n}\right)^{\otimes p}\left(\tilde{\mathbb{Y}}_{k}^{n}-\tilde{\mathbb{Y}}_{k-1}^{n}\right)^{\otimes q} \\
& -\frac{1}{2} \sum_{k=2}^{b_{n}-2}\left(\left(\tilde{\mathbb{Y}}_{k}^{n}-\tilde{\mathbb{Y}}_{k-1}^{n}\right)^{\otimes p}\left(\tilde{\mathbb{Y}}_{k+2}^{n}-\tilde{\mathbb{Y}}_{k+1}^{n}\right)^{\otimes q}\right. \\
& \left.\left.+\left(\tilde{\mathbb{Y}}_{k+2}^{n}-\tilde{\mathbb{Y}}_{k+1}^{n}\right)^{\otimes p}\left(\tilde{\mathbb{Y}}_{k}^{n}-\tilde{\mathbb{Y}}_{k-1}^{n}\right)^{\otimes q}\right)\right\}\left(\frac{T}{b_{n}}\right)^{-1}
\end{aligned}
$$

By the similar idea, we define

$$
\begin{aligned}
\hat{\gamma}_{n, 2}^{p q}=\frac{9}{8} \sum_{k=2}^{b_{n}-2} & \frac{1}{2}\left\{\left(\tilde{\mathbb{Y}}_{k+2}^{n}-\tilde{\mathbb{Y}}_{k+1}^{n}\right)^{\otimes p}-\left(\tilde{\mathbb{Y}}_{k}^{n}-\tilde{\mathbb{Y}}_{k-1}^{n}\right)^{\otimes p}\right\} \\
& \times\left\{\left(\tilde{\mathbb{Y}}_{k+2}^{n}-\tilde{\mathbb{Y}}_{k+1}^{n}\right)^{\otimes q}-\left(\tilde{\mathbb{Y}}_{k}^{n}-\tilde{\mathbb{Y}}_{k-1}^{n}\right)^{\otimes q}\right\}\left(\frac{T}{b_{n}}\right)^{-1} .
\end{aligned}
$$

\subsection{Kernel based $\Gamma$ estimator}

In the similar way in Hayashi and Yoshida [22, we define the kernel based estimator.

$$
\partial_{h}\left\{X^{\alpha}, X^{\beta}\right\}_{k}:=\sum_{l=(k-n(h)+1) \vee 2}^{k}\left(\Delta_{l} X^{\alpha} \Delta_{l} X^{\beta}\right) h^{-1} .
$$

where $h=h_{n}$ is a parameter satisfying $h_{n} \rightarrow 0$ and $h_{n}^{-1} b_{n}^{-1} \rightarrow 0$, and $n(h):=$ $\max _{m}\left\{t_{m} \leq h\right\}$.

$$
\begin{aligned}
\hat{\gamma}_{n, h}^{p q}=\frac{9}{8} \sum_{k=2}^{b_{n}} & \left\{\partial_{h}\left\{\tilde{Y}^{n, \alpha_{1}}, \tilde{Y}^{n, \alpha_{2}}\right\}_{k} \partial_{h}\left\{\tilde{Y}^{n, \beta_{1}}, \tilde{Y}^{n, \beta_{2}}\right\}_{k}\right. \\
& \left.+\partial_{h}\left\{\tilde{Y}^{n, \alpha_{1}}, \tilde{Y}^{n, \beta_{2}}\right\}_{k} \partial_{h}\left\{\tilde{Y}^{n, \beta_{1}}, \tilde{Y}^{n, \alpha_{2}}\right\}_{k}\right\}\left(\frac{T}{b_{n}}\right)
\end{aligned}
$$

\section{$3.3 \quad \Xi$ estimator}

Here, we define

$$
\hat{\Xi}_{n, *}^{1 / 2}=\left(\frac{1}{\sqrt{S_{n}^{11} S_{n}^{22}}}, \frac{-S_{n}^{12}}{2 \sqrt{\left(S_{n}^{11}\right)^{3} S_{n}^{22}}}, \frac{-S_{n}^{12}}{2 \sqrt{S_{n}^{11}\left(S_{n}^{22}\right)^{3}}}\right) \hat{\Gamma}_{n, *}^{1 / 2}
$$

and $\hat{\Xi}_{n, *}=\left(\hat{\Xi}_{n, *}^{1 / 2}\right)^{\otimes}$, for $*=1,2, h$.

\section{4 consistency of the estimators}

Theorem 3.1. (a) $\hat{\gamma}_{n, i}^{p q}=\gamma^{p q}+o_{p}(1)$ under $[A]$ and any one of $[B],\left[B^{\prime}\right]$ and $\left[B^{\sharp}\right],(i=1,2)$.
(b) $\hat{\gamma}_{n, i}^{p q}=\gamma^{p q}+O_{p}\left(b_{n}^{-1 / 2}\right)$ under $[A]$ and $\left[B^{\sharp^{\sharp}}\right],(i=1,2)$.
(c) $\hat{\gamma}_{n, i}^{p q}=\gamma^{p q}+O_{p}\left(b_{n}^{-1 / 2}\right)$ under $[A],[B]$ or $\left[B^{\prime}\right]$, and $[C](i=1,2)$. 
Theorem 3.2. (a) $\hat{\gamma}_{n, h}^{p q}=\gamma^{p q}+o_{p}(1)$ under $[A]$ and any one of $[B],\left[B^{\prime}\right]$ and $\left[B^{\sharp \sharp}\right]$.

(b) $\hat{\gamma}_{n, h}^{p q}=\gamma^{p q}+O_{p}\left(b_{n}^{-1 / 2}\right)+O_{p}\left(h_{n}\right)$ under $[A]$ and $\left[B^{\sharp}\right]$.

(c) $\hat{\gamma}_{n, h}^{p q}=\gamma^{p q}+O_{p}\left(b_{n}^{-1 / 2}\right)+O_{p}\left(h_{n}\right)$ under $[A]$, [B] or [B'], and [C].

Corollary 3.3. (a) $\hat{\Xi}_{n, i}=\Xi+o_{p}(1)$ under $[A]$ and any one of $[B],\left[B^{\prime}\right]$ and $\left[B^{\sharp}\right],(i=1,2)$.

(b) $\hat{\Xi}_{n, i}=\Xi+O_{p}\left(b_{n}^{-1 / 2}\right)$ under $[A]$ and $\left[B^{\sharp \sharp}\right],(i=1,2)$.

(c) $\hat{\Xi}_{n, i}=\Xi+O_{p}\left(b_{n}^{-1 / 2}\right)$ under $[A],[B]$ or $\left[B^{\prime}\right]$, and $[C](i=1,2)$.

Corollary 3.4. (a) $\hat{\Xi}_{n, h}=\Xi+o_{p}(1)$ under $[A]$ and any one of $[B],\left[B^{\prime}\right]$ and $\left[B^{\sharp \sharp}\right]$.

(b) $\hat{\Xi}_{n, h}=\Xi+O_{p}\left(b_{n}^{-1 / 2}\right)+O_{p}\left(h_{n}\right)$ under $[A]$ and $\left[B^{\sharp^{\sharp}}\right]$.

(c) $\hat{\Xi}_{n, h}=\Xi+O_{p}\left(b_{n}^{-1 / 2}\right)+O_{p}\left(h_{n}\right)$ under $[A]$, [B] or [B'], and [C].

\section{Proof}

\section{Proof of Theorem 3.1}

First, we approximate $\hat{\gamma}_{n, i}^{p q}(i=1,2)$ by the following quantities. Let

$$
\begin{aligned}
G_{n, 1}^{p q}=\frac{9}{8}\left\{\sum_{k=2}^{b_{n}}\left(\tilde{\chi}_{k}^{n}-\tilde{\chi}_{k-1}^{n}\right)^{\otimes p}\left(\tilde{\chi}_{k}^{n}-\tilde{\chi}_{k-1}^{n}\right)^{\otimes q}\right. \\
\quad-\frac{1}{2} \sum_{k=2}^{b_{n}-2}\left(\left(\tilde{\chi}_{k}^{n}-\tilde{\chi}_{k-1}^{n}\right)^{\otimes p}\left(\tilde{\chi}_{k+2}^{n}-\tilde{\chi}_{k+1}^{n}\right)^{\otimes q}\right. \\
\left.\left.\quad+\left(\tilde{\chi}_{k+2}^{n}-\tilde{\chi}_{k+1}^{n}\right)^{\otimes p}\left(\tilde{\chi}_{k}^{n}-\tilde{\chi}_{k-1}^{n}\right)^{\otimes q}\right)\right\}\left(\frac{T}{b_{n}}\right)^{-1}
\end{aligned}
$$

and

$$
\begin{aligned}
G_{n, 2}^{p q}=\frac{9}{8} \sum_{k=2}^{b_{n}-2} & \frac{1}{2}\left\{\left(\tilde{\chi}_{k+2}^{n}-\tilde{\chi}_{k+1}^{n}\right)^{\otimes p}-\left(\tilde{\chi}_{k}^{n}-\tilde{\chi}_{k-1}^{n}\right)^{\otimes p}\right\} \\
& \times\left\{\left(\tilde{\chi}_{k+2}^{n}-\tilde{\chi}_{k+1}^{n}\right)^{\otimes q}-\left(\tilde{\chi}_{k}^{n}-\tilde{\chi}_{k-1}^{n}\right)^{\otimes q}\right\}\left(\frac{T}{b_{n}}\right)^{-1} .
\end{aligned}
$$

where $\chi^{n}=\left(\chi^{n, 1}, \chi^{n, 2}\right)$ and $\chi_{t}^{n, \alpha}=\int_{0}^{t} a_{n} X_{s}^{\alpha} d s$.

Lemma 4.1. (a) $\hat{\gamma}_{n, i}^{p q}=G_{n, i}^{p q}+o_{p}(1)$ under any one of $[B],\left[B^{\prime}\right]$ and $\left[B^{\sharp}\right]$, $(i=1,2)$.

(b) $\hat{\gamma}_{n, i}^{p q}=G_{n, i}^{p q}+o_{p}\left(b_{n}^{-1 / 2}\right)$ under $\left[B^{\sharp \sharp}\right],(i=1,2)$.

Proof. Write $\left(V^{1} \otimes V^{2} \otimes \cdots \otimes V^{n}\right)_{i_{1}, i_{2}, \ldots, i_{n}}=V_{i_{1}}^{1} V_{i_{2}}^{2} \cdots V_{i_{n}}^{n}$ for vectors $V^{1}, V^{2}, \ldots, V^{n}$ and confirm $\left|V^{1} \otimes V^{2} \otimes \cdots \otimes V^{n}\right|=\left|V^{1}\right|\left|V^{2}\right| \cdots\left|V^{n}\right|$. Let $s_{k}=\left(\tilde{\mathbb{Y}}_{k}^{n}-\tilde{\mathbb{Y}}_{k-1}^{n}\right)^{\otimes}, t_{k}=$ $\left(\tilde{\chi}_{k}^{n}-\tilde{\chi}_{k-1}^{n}\right)^{\otimes}, \hat{\gamma}_{n, i}=\left(\hat{\gamma}_{n, i}^{\alpha_{1}, \beta_{1}, \alpha_{2}, \beta_{2}}\right)_{\alpha_{1}, \beta_{1}, \alpha_{2}, \beta_{2}=1,2}$, and $G_{n, i}=\left(G_{n, i}^{\alpha_{1}, \beta_{1}, \alpha_{2}, \beta_{2}}\right)_{\alpha_{1}, \beta_{1}, \alpha_{2}, \beta_{2}=1,2}$ 
$(i=1,2)$. It holds that

$$
\begin{aligned}
& \left(\frac{9}{8}\right)^{-1}\left|\hat{\gamma}_{n, 1}-G_{n, 1}\right| \\
& =\left(\frac{T}{b_{n}}\right)^{-1} \mid \sum_{k=2}^{b_{n}}\left(s_{k} \otimes s_{k}-t_{k} \otimes t_{k}\right) \\
& \quad-\frac{1}{2} \sum_{k=2}^{b_{n}-2}\left(\left(s_{k} \otimes s_{k+2}-t_{k} \otimes t_{k+2}\right)+\left(s_{k+2} \otimes s_{k}-t_{k+2} \otimes t_{k}\right)\right) \mid \\
& \leq\left(\frac{T}{b_{n}}\right)^{-1}\left\{\sum_{k=2}^{b_{n}}\left|s_{k} \otimes s_{k}-t_{k} \otimes t_{k}\right|\right. \\
& \left.\quad+\frac{1}{2} \sum_{k=2}^{b_{n}-2}\left|s_{k} \otimes s_{k+2}-t_{k} \otimes t_{k+2}\right|+\frac{1}{2} \sum_{k=2}^{b_{n}-2}\left|s_{k+2} \otimes s_{k}-t_{k+2} \otimes t_{k}\right|\right\}
\end{aligned}
$$

and

$$
\begin{aligned}
& \left(\frac{9}{8}\right)^{-1}\left|\hat{\gamma}_{n, 2}-G_{n, 2}\right| \\
& =\frac{1}{2}\left(\frac{T}{b_{n}}\right)^{-1} \mid \sum_{k=2}^{b_{n}-2}\left(s_{k+2} \otimes s_{k+2}-t_{k+2} \otimes t_{k+2}\right)-\left(s_{k+2} \otimes s_{k}-t_{k+2} \otimes t_{k}\right) \\
& \quad-\left(s_{k} \otimes s_{k+2}-t_{k} \otimes t_{k+2}\right)+\left(s_{k} \otimes s_{k}-t_{k} \otimes t_{k}\right) \mid \\
& \leq \frac{1}{2}\left(\frac{T}{b_{n}}\right)^{-1}\left\{\sum_{k=2}^{b_{n}-2}\left|s_{k+2} \otimes s_{k+2}-t_{k+2} \otimes t_{k+2}\right|+\sum_{k=2}^{b_{n}-2}\left|s_{k+2} \otimes s_{k}-t_{k+2} \otimes t_{k}\right|\right. \\
& \left.\quad+\sum_{k=2}^{b_{n}-2}\left|s_{k} \otimes s_{k+2}-t_{k} \otimes t_{k+2}\right|+\sum_{k=2}^{b_{n}-2}\left|s_{k} \otimes s_{k}-t_{k} \otimes t_{k}\right|\right\} .
\end{aligned}
$$


Now, let $\rho=0,1 / 2$ and see the typical term.

$$
\begin{aligned}
& b_{n}^{1+\rho} \sum_{k=2}^{b_{n}-2}\left|s_{k+2} \otimes s_{k}-t_{k+2} \otimes t_{k}\right| \\
& =b_{n}^{1+\rho} \sum_{k=2}^{b_{n}-2} \mid\left(s_{k+2}-t_{k+2}\right) \otimes\left(s_{k}-t_{k}\right) \\
& \quad+\left(s_{k+2}-t_{k+2}\right) \otimes t_{k}+t_{k+2} \otimes\left(s_{k}-t_{k}\right) \mid \\
& \leq b_{n}^{1+\rho} \sum_{k=2}^{b_{n}-2}\left|\left(s_{k+2}-t_{k+2}\right) \otimes\left(s_{k}-t_{k}\right)\right| \\
& \quad+\left|\left(s_{k+2}-t_{k+2}\right) \otimes t_{k}\right|+\left|t_{k+2} \otimes\left(s_{k}-t_{k}\right)\right| \\
& =b_{n}^{1+\rho} \sum_{k=2}^{b_{n}-2}\left|s_{k+2}-t_{k+2}\right|\left|s_{k}-t_{k}\right|+\left|s_{k+2}-t_{k+2}\right|\left|t_{k}\right|+\left|t_{k+2}\right|\left|s_{k}-t_{k}\right| \\
& \leq\left(b_{n}^{1 / 2+\rho / 2} \sum_{k=2}^{b_{n}-2}\left|s_{k+2}-t_{k+2}\right|\right)\left(b_{n}^{1 / 2+\rho / 2} \sum_{k=2}^{b_{n}-2}\left|s_{k}-t_{k}\right|\right) \\
& \quad+\left(b_{n}^{\rho} \sum_{k=2}^{b_{n}-2}\left|s_{k+2}-t_{k+2}\right|\right)\left(b_{n} \max _{k}\left|t_{k}\right|\right) \\
& \quad+\left(b_{n} \max _{k}\left|t_{k+2}\right|\right)\left(b_{n}^{\rho} \sum_{k=2}^{b_{n}-2}\left|s_{k}-t_{k}\right|\right) .
\end{aligned}
$$

Under $\left[\mathrm{B}^{\sharp}\right]$, the same argument of the proof of Lemma 1 (b) in [26] under the assumption $[\mathrm{B}]$ yields

$$
b_{n}^{3 / 4} \sum_{k=2}^{b_{n}}\left|s_{k}-t_{k}\right| \rightarrow^{p} 0 .
$$

Under any one of $[\mathrm{B}],\left[\mathrm{B}^{\prime}\right]$ and $\left[\mathrm{B}^{\sharp}\right]$, it holds that

$$
b_{n}^{1 / 2} \sum_{k=2}^{b_{n}}\left|s_{k}-t_{k}\right| \rightarrow^{p} 0
$$

by the proof of Lemma 1 (b) in [26. By inequality (4) in [26, and chebychev's inequality or Markov's inequality, we have

$$
b_{n} \max _{k}\left|t_{k}\right|=O_{p}(1)
$$

Therefore, the typical term converges to 0 in probability. The other terms are evaluated in the same way. We obtain the conclusion.

Proposition 4.2. $\hat{\gamma}_{n, i}^{p q}=G_{n, i}^{p q}+o_{p}\left(b_{n}^{-1 / 2}\right)$ under [B] or [B'], and $[C](i=1,2)$. 
Proof. We just confirm the convergence of the easiest term.

$$
\begin{aligned}
& b_{n}^{1+\rho} \sum_{k=2}^{b_{n}-2}\left|s_{k} \otimes s_{k}-t_{k} \otimes t_{k}\right| \\
& =b_{n}^{1+\rho} \sum_{k=2}^{b_{n}-2}\left|\left(s_{k}-t_{k}\right) \otimes\left(s_{k}-t_{k}\right)+\left(s_{k}-t_{k}\right) \otimes t_{k}+t_{k} \otimes\left(s_{k}-t_{k}\right)\right|
\end{aligned}
$$

By definition,

$$
\begin{aligned}
s_{k}-t_{k}= & \left(\tilde{\mathbb{M}}_{k}^{n}-\tilde{\mathbb{M}}_{k-1}^{n}\right)^{\otimes}+\left(\tilde{\mathbb{M}}_{k}^{n}-\tilde{\mathbb{M}}_{k-1}^{n}\right) \otimes\left(\tilde{\boldsymbol{\chi}}_{k}^{n}-\tilde{\boldsymbol{\chi}}_{k-1}^{n}\right) \\
& +\left(\tilde{\chi}_{k}^{n}-\tilde{\chi}_{k-1}^{n}\right) \otimes\left(\tilde{\mathbb{M}}_{k}^{n}-\tilde{\mathbb{M}}_{k-1}^{n}\right) .
\end{aligned}
$$

Therefore, it is enough to prove the following equations.

$$
\begin{array}{r}
b_{n}^{1+\rho} \sum_{k=2}^{b_{n}-2}\left(\tilde{\mathbb{M}}_{k}^{n}-\tilde{\mathbb{M}}_{k-1}^{n}\right)^{\otimes 3}=o_{p}(1) \\
b_{n}^{1+\rho} \sum_{k=2}^{b_{n}-2}\left(\tilde{\mathbb{M}}_{k}^{n}-\tilde{\mathbb{M}}_{k-1}^{n}\right)^{\otimes 2} \otimes\left(\tilde{\boldsymbol{\chi}}_{k}^{n}-\tilde{\boldsymbol{\chi}}_{k-1}^{n}\right)=o_{p}(1) \\
b_{n}^{1+\rho} \sum_{k=2}^{b_{n}-2}\left(\tilde{\mathbb{M}}_{k}^{n}-\tilde{\mathbb{M}}_{k-1}^{n}\right)^{\otimes} \otimes\left(\tilde{\boldsymbol{\chi}}_{k}^{n}-\tilde{\boldsymbol{\chi}}_{k-1}^{n}\right)^{\otimes}=o_{p}(1) \\
b_{n}^{1+\rho} \sum_{k=2}^{b_{n}-2}\left(\tilde{\mathbb{M}}_{k}^{n}-\tilde{\mathbb{M}}_{k-1}^{n}\right) \otimes\left(\tilde{\boldsymbol{\chi}}_{k}^{n}-\tilde{\boldsymbol{\chi}}_{k-1}^{n}\right)^{\otimes 2}=o_{p}(1),
\end{array}
$$

where for vector $V, V^{\otimes}=V \otimes V, V^{\otimes 2}=V \otimes V \otimes V, V^{\otimes 3}=V \otimes V \otimes V \otimes V$. By assumption $[\mathrm{C}]$, it holds that

$$
\begin{aligned}
E\left[\left|b_{n}^{1+\rho} \sum_{k=2}^{b_{n}-2}\left(\tilde{\mathbb{M}}_{k}^{n}-\tilde{\mathbb{M}}_{k-1}^{n}\right)^{\otimes 3}\right|\right] & \leq E\left[b_{n}^{1+\rho} \sum_{k=2}^{b_{n}-2}\left|\tilde{\mathbb{M}}_{k}^{n}-\tilde{\mathbb{M}}_{k-1}^{n}\right|^{4}\right] \\
& \leq 16 E\left[b_{n}^{1+\rho} \sum_{k=2}^{b_{n}-2}\left|\tilde{\mathbb{M}}_{k}^{n}\right|^{4}\right] \\
& =O\left(b_{n}^{4+\rho} a_{n}^{-2}\right)=o(1) .
\end{aligned}
$$

Lemma 1 (b) and inequality (4) in [26] yields

$$
\begin{aligned}
& \left|b_{n}^{1+\rho} \sum_{k=2}^{b_{n}-2}\left(\tilde{\mathbb{M}}_{k}^{n}-\tilde{\mathbb{M}}_{k-1}^{n}\right)^{\otimes} \otimes\left(\tilde{\chi}_{k}^{n}-\tilde{\chi}_{k-1}^{n}\right)^{\otimes}\right| \\
& \leq\left(b_{n}^{\rho} \sum_{k=2}^{b_{n}-2}\left|\tilde{\mathbb{M}}_{k}^{n}-\tilde{\mathbb{M}}_{k-1}^{n}\right|^{2}\right)\left(b_{n}\left|\tilde{\chi}_{k}^{n}-\tilde{\boldsymbol{\chi}}_{k-1}^{n}\right|^{2}\right)=o_{p}(1) O_{p}(1)=o_{p}(1) .
\end{aligned}
$$


and

$$
\begin{aligned}
& \left|b_{n}^{1+\rho} \sum_{k=2}^{b_{n}-2}\left(\tilde{\mathbb{M}}_{k}^{n}-\tilde{\mathbb{M}}_{k-1}^{n}\right) \otimes\left(\tilde{\chi}_{k}^{n}-\tilde{\chi}_{k-1}^{n}\right)^{\otimes \otimes}\right| \\
& \leq\left|b_{n}^{\rho} \sum_{k=2}^{b_{n}-2}\left(\tilde{\mathbb{M}}_{k}^{n}-\tilde{\mathbb{M}}_{k-1}^{n}\right) \otimes\left(\tilde{\chi}_{k}^{n}-\tilde{\chi}_{k-1}^{n}\right)\right|\left|b_{n}\left(\tilde{\chi}_{k}^{n}-\tilde{\chi}_{k-1}^{n}\right)^{\otimes}\right| \\
& =o_{p}(1) O_{p}(1)=o_{p}(1) .
\end{aligned}
$$

From the above inequalities, it is derived that

$$
\begin{aligned}
& \left|b_{n}^{1+\rho} \sum_{k=2}^{b_{n}-2}\left(\tilde{\mathbb{M}}_{k}^{n}-\tilde{\mathbb{M}}_{k-1}^{n}\right)^{\otimes 2} \otimes\left(\tilde{\chi}_{k}^{n}-\tilde{\chi}_{k-1}^{n}\right)\right| \\
& \leq\left|b_{n}^{1+\rho} \sum_{k=2}^{b_{n}-2}\left(\tilde{\mathbb{M}}_{k}^{n}-\tilde{\mathbb{M}}_{k-1}^{n}\right)^{\otimes 3}\right|^{1 / 2} \\
& \times\left|b_{n}^{1+\rho} \sum_{k=2}^{b_{n}-2}\left(\tilde{\mathbb{M}}_{k}^{n}-\tilde{\mathbb{M}}_{k-1}^{n}\right)^{\otimes} \otimes\left(\tilde{\boldsymbol{\chi}}_{k}^{n}-\tilde{\boldsymbol{\chi}}_{k-1}^{n}\right)^{\otimes}\right|^{1 / 2} \\
& =o_{p}(1) o_{p}(1)=o_{p}(1) \text {. }
\end{aligned}
$$

Now, we let

$$
\begin{aligned}
& A_{k}^{\alpha}=X_{t_{k-2}}^{\alpha 1} \int_{I_{k-1}} H_{k}(s) d w_{s}+X_{t_{k-2}}^{\alpha 1} \int_{I_{k}} K_{k}(s) d w_{s} \\
& B_{k}^{\alpha}=\frac{1}{h_{k}} \int_{I_{k}} \int_{t_{k-2}}^{t}\left(X_{s}^{\alpha 1}-X_{t_{k-2}}^{\alpha 1}\right) d w_{s} d t-\frac{1}{h_{k-1}} \int_{I_{k-1}} \int_{t_{k-2}}^{t}\left(X_{s}^{\alpha 1}-X_{t_{k-2}}^{\alpha 1}\right) d w_{s} d t \\
& C_{k}^{\alpha}=\frac{1}{h_{k}} \int_{I_{k}} \int_{t_{k-2}}^{t} X_{t_{k-2}}^{\alpha 0} d s d t-\frac{1}{h_{k-1}} \int_{I_{k-1}} \int_{t_{k-2}}^{t} X_{t_{k-2}}^{\alpha 0} d s d t \\
& D_{k}^{\alpha}=\frac{1}{h_{k}} \int_{I_{k}} \int_{t_{k-2}}^{t}\left(X_{s}^{\alpha 0}-X_{t_{k-2}}^{\alpha 0}\right) d s d t-\frac{1}{h_{k-1}} \int_{I_{k-1}} \int_{t_{k-2}}^{t}\left(X_{s}^{\alpha 0}-X_{t_{k-2}}^{\alpha 0}\right) d s d t .
\end{aligned}
$$

By Lemma 2 and inequality (4) in [26, we have

$$
\begin{aligned}
& G_{n, 1}^{p q}= \frac{9}{8}\left\{\sum_{k=2}^{b_{n}}\left(A_{k}^{\alpha_{1}} A_{k}^{\beta_{1}}\right)\left(A_{k}^{\alpha_{2}} A_{k}^{\beta_{2}}\right)\right. \\
&-\frac{1}{2} \sum_{k=2}^{b_{n}-2}\left(\left(A_{k}^{\alpha_{1}} A_{k}^{\beta_{1}}\right)\left(A_{k+2}^{\alpha_{2}} A_{k+2}^{\beta_{2}}\right)\right. \\
&\left.\left.\quad+\left(A_{k+2}^{\alpha_{1}} A_{k+2}^{\beta_{1}}\right)\left(A_{k}^{\alpha_{2}} A_{k}^{\beta_{2}}\right)\right)\right\}\left(\frac{T}{b_{n}}\right)^{-1}+o_{p}\left(b_{n}^{-1 / 2}\right) \\
&=\mathcal{R}_{n, 1}^{1, p q}+o_{p}\left(b_{n}^{-1 / 2}\right)
\end{aligned}
$$


and

$$
\begin{aligned}
G_{n, 2}^{p q}=\frac{9}{8} \sum_{k=2}^{b_{n}-2} \frac{1}{2}\left\{\left(A_{k+2}^{\alpha_{1}} A_{k+2}^{\beta_{1}}\right)\left(A_{k+2}^{\alpha_{2}} A_{k+2}^{\beta_{2}}\right)\right. \\
-\left(A_{k+2}^{\alpha_{1}} A_{k+2}^{\beta_{1}}\right)\left(A_{k}^{\alpha_{2}} A_{k}^{\beta_{2}}\right) \\
-\left(A_{k}^{\alpha_{1}} A_{k}^{\beta_{1}}\right)\left(A_{k+2}^{\alpha_{2}} A_{k+2}^{\beta_{2}}\right) \\
\left.+\left(A_{k}^{\alpha_{1}} A_{k}^{\beta_{1}}\right)\left(A_{k}^{\alpha_{2}} A_{k}^{\beta_{2}}\right)\right\}\left(\frac{T}{b_{n}}\right)^{-1}+o_{p}\left(b_{n}^{-1 / 2}\right) \\
=\mathcal{R}_{n, 2}^{1, p q}+o_{p}\left(b_{n}^{-1 / 2}\right)
\end{aligned}
$$

Here, we write

$$
q_{k}^{2, \alpha \beta}=X_{t_{k-2}}^{\alpha 1} \cdot X_{t_{k-2}}^{\beta 1} \frac{h_{k-1}}{3}+X_{t_{k-2}}^{\alpha 1} \cdot X_{t_{k-2}}^{\beta 1} \frac{h_{k}}{3}
$$

and

$$
\begin{aligned}
q_{k}^{3, \alpha \beta}=2\left(\mathbb{X}_{t_{k-2}}^{1}\right)^{\tilde{\otimes}(\alpha, \beta)} & \cdot\left(\int_{I_{k-1}} \int_{t_{k-2}}^{t} H_{k}(s) d w_{s} \otimes H_{k}(t) d w_{t}\right. \\
& +\int_{I_{k}} \int_{t_{k-1}}^{t} K_{k}(s) d w_{s} \otimes K_{k}(t) d w_{t} \\
& \left.+\int_{I_{k-1}} H_{k}(s) d w_{s} \otimes \int_{I_{k}} K_{k}(s) d w_{s}\right) .
\end{aligned}
$$

Then, we have

$$
\begin{aligned}
\mathcal{R}_{n, 1}^{1, p q}=\frac{9}{8}\left\{\sum_{k=2}^{b_{n}}\left(q_{k}^{2, p}+q_{k}^{3, p}\right)\left(q_{k}^{2, q}+q_{k}^{3, q}\right)\right. & \\
-\frac{1}{2} \sum_{k=2}^{b_{n}-2} & \left(\left(q_{k}^{2, p}+q_{k}^{3, p}\right)\left(q_{k+2}^{2, q}+q_{k+2}^{3, q}\right)\right. \\
& \left.\left.\quad\left(q_{k+2}^{2, p}+q_{k+2}^{3, p}\right)\left(q_{k}^{2, q}+q_{k}^{3, q}\right)\right)\right\}\left(\frac{T}{b_{n}}\right)^{-1}
\end{aligned}
$$

and

$$
\begin{aligned}
\mathcal{R}_{n, 2}^{1, p q}=\frac{9}{8} \sum_{k=2}^{b_{n}-2} \frac{1}{2}\left\{\left(q_{k+2}^{2, p}\right.\right. & \left.+q_{k+2}^{3, p}\right)\left(q_{k+2}^{2, p}+q_{k+2}^{3, p}\right) \\
& -\left(q_{k+2}^{2, p}+q_{k+2}^{3, p}\right)\left(q_{k}^{2, p}+q_{k}^{3, p}\right) \\
& -\left(q_{k}^{2, p}+q_{k}^{3, p}\right)\left(q_{k+2}^{2, p}+q_{k+2}^{3, p}\right) \\
& \left.+\left(q_{k}^{2, p}+q_{k}^{3, p}\right)\left(q_{k}^{2, p}+q_{k}^{3, p}\right)\right\}\left(\frac{T}{b_{n}}\right)^{-1}
\end{aligned}
$$

by Itô's formula and simple calculus. We only see the typical terms. We have

$$
\left(\frac{T}{b_{n}}\right)^{-1} \sum_{k=2}^{b_{n}} q_{k}^{2, p} q_{k}^{2, q}=\frac{4}{9} \sum_{k=2}^{b_{n}}\left(X_{t_{k-2}}^{\alpha_{1} 1} \cdot X_{t_{k-2}}^{\beta_{1} 1}\right)\left(X_{t_{k-2}}^{\alpha_{2} 1} \cdot X_{t_{k-2}}^{\beta_{2} 1}\right)\left(\frac{T}{b_{n}}\right)
$$




$$
\left(\frac{T}{b_{n}}\right)^{-1} \sum_{k=2}^{b_{n}} q_{k}^{2, p} q_{k}^{3, q}=\sum_{k=2}^{b_{n}} \frac{2}{3}\left(X_{t_{k-2}}^{\alpha_{1} 1} \cdot X_{t_{k-2}}^{\beta_{1} 1}\right) q_{k}^{3, q}=O_{p}\left(b_{n}^{-1 / 2}\right)
$$

and

$$
\left(\frac{T}{b_{n}}\right)^{-1} \sum_{k=2}^{b_{n}-2} q_{k+2}^{3, p} q_{k}^{3, q}=O_{p}\left(b_{n}^{-1 / 2}\right) .
$$

By Lemma 4 in [26] and simple calculus, it holds that

$$
\begin{aligned}
& \left(\frac{T}{b_{n}}\right)^{-1} \sum_{k=2}^{b_{n}} q_{k}^{3, p} q_{k}^{3, q} \\
& =\left(\frac{T}{b_{n}}\right)^{-1} \sum_{k=2}^{b_{n}}\left\{\sum_{i, j=1}^{r}\left(X_{i, t_{k-2}}^{\alpha_{1} 1} X_{j, t_{k-2}}^{\beta_{1} 1}+X_{j, t_{k-2}}^{\alpha_{1} 1} X_{i, t_{k-2}}^{\beta_{1} 1}\right)\right. \\
& \times\left(\int_{I_{k-1}} \int_{t_{k-2}}^{t} H_{k}(s) d w_{s}^{i} H_{k}(t) d w_{t}^{j}\right. \\
& +\int_{I_{k}} \int_{t_{k-1}}^{t} K_{k}(s) d w_{s}^{i} K_{k}(t) d w_{t}^{j} \\
& \left.\left.+\int_{I_{k-1}} H_{k}(s) d w_{s}^{i} \int_{I_{k}} K_{k}(s) d w_{s}^{j}\right)\right\} \\
& \times\left\{\sum_{i, j=1}^{r}\left(X_{i, t_{k-2}}^{\alpha_{2} 1} X_{j, t_{k-2}}^{\beta_{2} 1}+X_{j, t_{k-2}}^{\alpha_{2} 1} X_{i, t_{k-2}}^{\beta_{2} 1}\right)\right. \\
& \times\left(\int_{I_{k-1}} \int_{t_{k-2}}^{t} H_{k}(s) d w_{s}^{i} H_{k}(t) d w_{t}^{j}\right. \\
& +\int_{I_{k}} \int_{t_{k-1}}^{t} K_{k}(s) d w_{s}^{i} K_{k}(t) d w_{t}^{j} \\
& \left.\left.+\int_{I_{k-1}} H_{k}(s) d w_{s}^{i} \int_{I_{k}} K_{k}(s) d w_{s}^{j}\right)\right\} \\
& =\left(\frac{T}{b_{n}}\right)^{-1} \sum_{k=2}^{b_{n}}\left\{\sum_{i, j=1}^{r}\left(X_{i, t_{k-2}}^{\alpha_{1} 1} X_{j, t_{k-2}}^{\beta_{1} 1}+X_{j, t_{k-2}}^{\alpha_{1} 1} X_{i, t_{k-2}}^{\beta_{1} 1}\right)\right. \\
& \left.\times\left(X_{i, t_{k-2}}^{\alpha_{2} 1} X_{j, t_{k-2}}^{\beta_{2} 1}+X_{j, t_{k-2}}^{\alpha_{2} 1} X_{i, t_{k-2}}^{\beta_{2} 1}\right)\right\} \\
& \times\left\{\int_{0}^{T} \int_{0}^{t}\left(\tilde{H}_{k}(s)\right)^{2} d s\left(\tilde{H}_{k}(t)\right)^{2} d t\right. \\
& +\int_{0}^{T} \int_{0}^{t}\left(\tilde{K}_{k}(s)\right)^{2} d s\left(\tilde{K}_{k}(t)\right)^{2} d t \\
& \left.+\int_{0}^{T}\left(\tilde{H}_{k}(s)\right)^{2} d s \int_{0}^{T}\left(\tilde{H}_{k}(s)\right)^{2} d s\right\}+O_{p}\left(b_{n}^{-1 / 2}\right) \\
& =\frac{4}{9} \sum_{k=2}^{b_{n}}\left\{\left(X_{t_{k-2}}^{\alpha_{1} 1} \cdot X_{t_{k-2}}^{\alpha_{2} 1}\right)\left(X_{t_{k-2}}^{\beta_{1} 1} \cdot X_{t_{k-2}}^{\beta_{2} 1}\right)\right. \\
& \left.+\left(X_{t_{k-2}}^{\alpha_{1} 1} \cdot X_{t_{k-2}}^{\beta_{2} 1}\right)\left(X_{t_{k-2}}^{\beta_{1} 1} \cdot X_{t_{k-2}}^{\alpha_{2} 1}\right)\right\}\left(\frac{T}{b_{n}}\right)+O_{p}\left(b_{n}^{-1 / 2}\right),
\end{aligned}
$$


where $\tilde{H}_{k}(s)=1_{I_{k-1}}(s) H_{k}(s)$ and $\tilde{K}_{k}(s)=1_{I_{k}}(s) K_{k}(s)$. Therefore, we have

$$
\begin{aligned}
\mathcal{R}_{n, 1}^{1, p q}=\frac{9}{8}\{ & \frac{4}{9} \sum_{k=2}^{b_{n}}\left(X_{t_{k-2}}^{\alpha_{1} 1} \cdot X_{t_{k-2}}^{\beta_{1} 1}\right)\left(X_{t_{k-2}}^{\alpha_{2} 1} \cdot X_{t_{k-2}}^{\beta_{2} 1}\right)\left(\frac{T}{b_{n}}\right) \\
+ & \frac{4}{9} \sum_{k=2}^{b_{n}}\left\{\left(X_{t_{k-2}}^{\alpha_{1} 1} \cdot X_{t_{k-2}}^{\alpha_{2} 1}\right)\left(X_{t_{k-2}}^{\beta_{1} 1} \cdot X_{t_{k-2}}^{\beta_{2} 1}\right)\right. \\
& \left.+\left(X_{t_{k-2}}^{\alpha_{1} 1} \cdot X_{t_{k-2}}^{\beta_{2} 1}\right)\left(X_{t_{k-2}}^{\beta_{1} 1} \cdot X_{t_{k-2}}^{\alpha_{2} 1}\right)\right\}\left(\frac{T}{b_{n}}\right) \\
- & \frac{1}{2}\left\{\frac{4}{9} \sum_{k=2}^{b_{n}-2}\left(X_{t_{k-2}}^{\alpha_{1} 1} \cdot X_{t_{k-2}}^{\beta_{1} 1}\right)\left(X_{t_{k}}^{\alpha_{2} 1} \cdot X_{t_{k}}^{\beta_{2} 1}\right)\left(\frac{T}{b_{n}}\right)\right. \\
& \left.\left.+\frac{4}{9} \sum_{k=2}^{b_{n}-2}\left(X_{t_{k}}^{\alpha_{1} 1} \cdot X_{t_{k}}^{\beta_{1} 1}\right)\left(X_{t_{k-2}}^{\alpha_{2} 1} \cdot X_{t_{k-2}}^{\beta_{2} 1}\right)\left(\frac{T}{b_{n}}\right)\right\}\right\} \\
+ & O_{p}\left(b_{n}^{-1 / 2}\right) \\
=\gamma^{p q} & +o_{p}\left(b_{n}^{-1 / 2}\right)+O_{p}\left(b_{n}^{-1 / 2}\right)
\end{aligned}
$$

and

$$
\begin{aligned}
\mathcal{R}_{n, 2}^{1, p q}=\frac{9}{16}\{ & \frac{4}{9} \sum_{k=2}^{b_{n}-2}\left(X_{t_{k}}^{\alpha_{1} 1} \cdot X_{t_{k}}^{\beta_{1} 1}\right)\left(X_{t_{k}}^{\alpha_{2} 1} \cdot X_{t_{k}}^{\beta_{2} 1}\right)\left(\frac{T}{b_{n}}\right) \\
& +\frac{4}{9} \sum_{k=2}^{b_{n}-2}\left\{\left(X_{t_{k-2}}^{\alpha_{1} 1} \cdot X_{t_{k-2}}^{\alpha_{2} 1}\right)\left(X_{t_{k-2}}^{\beta_{1} 1} \cdot X_{t_{k-2}}^{\beta_{2} 1}\right)\right. \\
& \left.+\left(X_{t_{k-2}}^{\alpha_{1} 1} \cdot X_{t_{k-2}}^{\beta_{2} 1}\right)\left(X_{t_{k-2}}^{\beta_{1} 1} \cdot X_{t_{k-2}}^{\alpha_{2} 1}\right)\right\}\left(\frac{T}{b_{n}}\right) \\
& -\frac{4}{9} \sum_{k=2}^{b_{n}-2}\left(X_{t_{k}}^{\alpha_{1} 1} \cdot X_{t_{k}}^{\beta_{1} 1}\right)\left(X_{t_{k-2}}^{\alpha_{2} 1} \cdot X_{t_{k-2}}^{\beta_{2} 1}\right)\left(\frac{T}{b_{n}}\right) \\
& -\frac{4}{9} \sum_{k=2}^{b_{n}-2}\left(X_{t_{k-2}}^{\alpha_{1} 1} \cdot X_{t_{k-2}}^{\beta_{1} 1}\right)\left(X_{t_{k}}^{\alpha_{2} 1} \cdot X_{t_{k}}^{\beta_{2} 1}\right)\left(\frac{T}{b_{n}}\right) \\
& +\frac{4}{9} \sum_{k=2}^{b_{n}-2}\left(X_{t_{k-2}}^{\alpha_{1} 1} \cdot X_{t_{k-2}}^{\beta_{1} 1}\right)\left(X_{t_{k-2}}^{\alpha_{2} 1} \cdot X_{t_{k-2}}^{\beta_{2} 1}\right)\left(\frac{T}{b_{n}}\right) \\
& +\frac{4}{9} \sum_{k=2}^{b_{n}-2}\left\{\left(X_{t_{k-2}}^{\alpha_{1} 1} \cdot X_{t_{k-2}}^{\alpha_{2} 1}\right)\left(X_{t_{k-2}}^{\beta_{1} 1} \cdot X_{t_{k-2}}^{\beta_{2} 1}\right)\right. \\
+ & O_{p}\left(b_{n}^{-1 / 2}\right) . \\
=\gamma^{p q} & \left.+O_{p}\left(b_{n}^{-1 / 2}\right)+O_{t_{k-2}}^{\alpha_{1} 1} \cdot X_{t_{k-2}}^{\beta_{2} 1}\right)\left({b_{n}}_{t_{k-2}}^{\beta_{1} 1}\right) .
\end{aligned}
$$

The last $o_{p}\left(b_{n}^{-1 / 2}\right) \mathrm{s}$ are derived from the proof of Lemma 3 in [26] and simple calculus. 


\section{Proof of Theorem 3.2}

In the similar way to the proof of Therem 3.1, let

$$
\begin{aligned}
G_{n, h}^{p q}=\frac{9}{8} \sum_{k=2}^{b_{n}}\{ & \partial_{h}\left\{\tilde{\chi}^{n, \alpha_{1}}, \tilde{\chi}^{n, \alpha_{2}}\right\}_{k} \partial_{h}\left\{\tilde{\chi}^{n, \beta_{1}}, \tilde{\chi}^{n, \beta_{2}}\right\}_{k} \\
& \left.+\partial_{h}\left\{\tilde{\chi}^{n, \alpha_{1}}, \tilde{\chi}^{n, \beta_{2}}\right\}_{k} \partial_{h}\left\{\tilde{\chi}^{n, \beta_{1}}, \tilde{\chi}^{n, \alpha_{2}}\right\}_{k}\right\}\left(\frac{T}{b_{n}}\right) .
\end{aligned}
$$

Lemma 4.3. (a) $\hat{\gamma}_{n, h}^{p q}=G_{n, h}^{p q}+o_{p}(1)$ under any one of $[B],\left[B^{\prime}\right]$ and $\left[B^{\sharp \sharp}\right]$.

(b) $\hat{\gamma}_{n, h}^{p q}=G_{n, h}^{p q}+o_{p}\left(b_{n}^{-1 / 2}\right)$ under $\left[B^{\sharp \sharp}\right]$.

Proof. We write

$$
\check{s}_{k, h}:=\sum_{l=(k-n(h)+1) \vee 2}^{k} s_{l} h^{-1}, \quad \check{t}_{k, h}:=\sum_{l=(k-n(h)+1) \vee 2}^{k} t_{l} h^{-1},
$$

$\hat{\gamma}_{n, h}=\left(\hat{\gamma}_{n, h}^{p q}\right)_{p, q=(1,2),(2,1),(1,1),(2,2)}$, and $G_{n, h}=\left(G_{n, h}^{p q}\right)_{p, q=(1,2),(2,1),(1,1),(2,2)}$. It holds that

$$
\begin{aligned}
& b_{n}^{\rho}\left(\frac{9}{8}\right)^{-1}\left|\hat{\gamma}_{n, h}-G_{n, h}\right| \\
& =b_{n}^{\rho} 2\left|\sum_{k=2}^{b_{n}}\left(\check{s}_{k, h} \otimes \check{s}_{k, h}-\check{t}_{k, h} \otimes \check{t}_{k, h}\right)\right|\left(\frac{T}{b_{n}}\right) \\
& =b_{n}^{\rho} 2 \mid \sum_{k=2}^{b_{n}}\left(\check{s}_{k, h}-\check{t}_{k, h}\right) \otimes\left(\check{s}_{k, h}-\check{t}_{k, h}\right) \\
& \quad+\left(\check{s}_{k, h}-\check{t}_{k, h}\right) \otimes \check{t}_{k, h}+\check{t}_{k, h} \otimes\left(\check{s}_{k, h}-\check{t}_{k, h}\right) \mid\left(\frac{T}{b_{n}}\right) \\
& \leq b_{n}^{\rho} 2 \sum_{k=2}^{b_{n}}\left\{\left|\left(\check{s}_{k, h}-\check{t}_{k, h}\right) \otimes\left(\check{s}_{k, h}-\check{t}_{k, h}\right)\right|\right. \\
& \left.=b_{n}^{\rho} 2 \sum_{k=2}^{b_{n}}\left|\check{s}_{k, h}-\check{t}_{k, h}\right|^{2}\left(\frac{T}{b_{n}}\right)+\check{t}_{k, h}\right) \otimes \check{t}_{k, h}|+| \check{t}_{k, h} \otimes \sum_{k=2}^{b_{n}}\left|\check{t}_{k, h}\right|\left|\check{s}_{k, h}-\check{t}_{k, h}\right|\left(\frac{T}{b_{n}}\right) \\
& \left.\quad+4\left\{\check{t}_{k, h}\right) \mid\right\}\left(\frac{T}{b_{n}}\right) \\
& \quad 2\left\{b_{n}^{\rho / 2}\left(\frac{T}{b_{n}}\right)^{-1 / 2} \sum_{k=2}^{b_{n}}\left|\check{s}_{k, h}-\check{t}_{k, h}\right|\left(\frac{T}{b_{n}}\right)\right\} \\
& \left.\quad \check{t}_{k, h} \mid\right\}\left\{b_{n}^{\rho} \sum_{k=2}^{b_{n}}\left|\check{s}_{k, h}-\check{t}_{k, h}\right|\left(\frac{T}{b_{n}}\right)\right\} .
\end{aligned}
$$

We obtain the conclusion from the inequalities

$$
\sum_{k=2}^{b_{n}}\left|\check{s}_{k, h}-\check{t}_{k, h}\right|\left(\frac{T}{b_{n}}\right) \leq \sum_{k=2}^{b_{n}} \sum_{l=(k-n(h)+1) \vee 2}^{k}\left|s_{l}-t_{l}\right| h^{-1}\left(\frac{T}{b_{n}}\right) \leq \sum_{k=2}^{b_{n}}\left|s_{k}-t_{k}\right|,
$$




$$
\max _{k}\left|\check{t}_{k, h}\right| \leq \max _{k}\left|t_{k}\right|
$$

and the proof of Lemma 4.1

Proposition 4.4. $\hat{\gamma}_{n, h}^{p q}=G_{n, h}^{p q}+o_{p}\left(b_{n}^{-1 / 2}\right)$ under $[B]$ or $\left[B^{\prime}\right]$, and $[C]$.

Proof. The conclusion is derived from the proof of Lemma 4.3, that of Proposition 4.2, and the inequality

$$
b_{n}^{\rho} \sum_{k=2}^{b_{n}}\left|\check{s}_{k, h}-\check{t}_{k, h}\right|^{2}\left(\frac{T}{b_{n}}\right) \leq b_{n}^{\rho} \sum_{k=2}^{b_{n}}\left|s_{k}-t_{k}\right|^{2}\left(\frac{T}{b_{n}}\right)^{-1} .
$$

Lemma 4.5. $b_{n}^{\rho} \sum_{l=(k-n(h)+1) \vee 2}^{k} F_{l} G_{l} h^{-1} \rightarrow^{p} 0$ for all pairs of $\left(F_{l} G_{l}\right) \in\left\{A_{k}^{\alpha}, B_{k}^{\alpha}, C_{k}^{\alpha}, D_{k}^{\alpha} ; \alpha=1,2\right\}^{2} \backslash\left\{\left(A_{l}^{\alpha}, A_{l}^{\beta}\right) ; \alpha, \beta=1,2\right\}$.

The proof of this lemma is very similar to proof of Lemma 2 in [26].

Proof. Let $\mathcal{Q}_{n, h}=b_{n}^{\rho} \sum_{l=(k-n(h)+1) \vee 2}^{k} F_{l} G_{l} h^{-1}$. For $F_{l}=A_{l}^{\alpha}$ and $G_{l}=C_{l}^{\beta}$, $E\left[\left(\left|\mathcal{Q}_{n, h}\right|\right)^{2}\right]=O\left(b_{n}^{2 \rho} n(h) h^{-2} b_{n}^{-3}\right)=O\left(h^{-1} b_{n}^{2 \rho-2}\right)=o(1)$. For $F_{l}=A_{l}^{\alpha}$ and $G_{l}=D_{l}^{\beta}, E\left[\left|\mathcal{Q}_{n, h}\right|\right]=O\left(b_{n}^{\rho} n(h) h^{-1} b_{n}^{-3 / 2}\right) o(1)=o\left(b_{n}^{\rho-1 / 2}\right)=o(1)$. For $F_{l}=A_{l}^{\alpha}$ and $G_{l}=B_{l}^{\beta}, E\left[\left(\left|\mathcal{Q}_{n, h}\right|\right)^{2}\right]=O\left(b_{n}^{2 \rho} n(h) h^{-2} b_{n}^{-3}\right)=O\left(h^{-1} b_{n}^{2 \rho-2}\right)=o(1)$.

Now, let

$$
Q_{k, h}^{1, \alpha \beta}=\sum_{l=(k-n(h)+1) \vee 2}^{k} A_{l}^{\alpha} A_{l}^{\beta} h^{-1} .
$$

By Lemma 4.5 and (4) (esp. $Q_{k, h}^{1, \alpha \beta}=O_{p}(1)$ ), it holds that

$$
\begin{aligned}
G_{n, h}^{p q} & =\frac{9}{8} \sum_{k=2}^{b_{n}}\left(Q_{k, h}^{1, \alpha_{1} \alpha_{2}} Q_{k, h}^{1, \beta_{1} \beta_{2}}+Q_{k, h}^{1, \alpha_{1} \beta_{2}} Q_{k, h}^{1, \beta_{1} \alpha_{2}}\right)\left(\frac{T}{b_{n}}\right)+o_{p}\left(b_{n}^{-1 / 2}\right) \\
& =: \mathcal{R}_{n, h}^{1, p q}+o_{p}\left(b_{n}^{-1 / 2}\right) .
\end{aligned}
$$


By recombination of the multiple summation,

$$
\begin{aligned}
& \mathcal{R}_{n, h}^{1, p q}=\frac{9}{8} \sum_{l=2}^{b_{n}-n(h)}\left\{n(h)\left(q_{l}^{1, \alpha_{1} \alpha_{2}} q_{l}^{1, \beta_{1} \beta_{2}}+q_{l}^{1, \alpha_{1} \beta_{2}} q_{l}^{1, \beta_{1} \alpha_{2}}\right)\right. \\
& +(n(h)-1)\left(q_{l}^{1, \alpha_{1} \alpha_{2}} q_{l+1}^{1, \beta_{1} \beta_{2}}+q_{l}^{1, \alpha_{1} \beta_{2}} q_{l+1}^{1, \beta_{1} \alpha_{2}}\right. \\
& \left.+q_{l+1}^{1, \alpha_{1} \alpha_{2}} q_{l}^{1, \beta_{1} \beta_{2}}+q_{l+1}^{1, \alpha_{1} \beta_{2}} q_{l}^{1, \beta_{1} \alpha_{2}}\right) \\
& +\sum_{k=2}^{n(h)-1}(n(h)-k)\left(q_{l}^{1, \alpha_{1} \alpha_{2}} q_{l+k}^{1, \beta_{1} \beta_{2}}+q_{l}^{1, \alpha_{1} \beta_{2}} q_{l+k}^{1, \beta_{1} \alpha_{2}}\right. \\
& \left.\left.+q_{l+k}^{1, \alpha_{1} \alpha_{2}} q_{l}^{1, \beta_{1} \beta_{2}}+q_{l+k}^{1, \alpha_{1} \beta_{2}} q_{l}^{1, \beta_{1} \alpha_{2}}\right)\right\} h_{n}^{-2}\left(\frac{T}{b_{n}}\right) \\
& +\frac{9}{8} \sum_{m=b_{n}-n(h)+1}^{b_{n}}\left\{\left(\sum_{l=b_{n}-n(h)+1}^{m} q_{l}^{1, \alpha_{1} \alpha_{2}}\right)\left(\sum_{l=b_{n}-n(h)+1}^{m} q_{l}^{1, \beta_{1} \beta_{2}}\right)\right. \\
& \left.+\left(\sum_{l=b_{n}-n(h)+1}^{m} q_{l}^{1, \alpha_{1} \beta_{2}}\right)\left(\sum_{l=b_{n}-n(h)+1}^{m} q_{l}^{1, \beta_{1} \alpha_{2}}\right)\right\} h_{n}^{-2}\left(\frac{T}{b_{n}}\right) \\
& =: \mathcal{B}_{n, h}^{1, p q}+\mathcal{T}_{n, h}^{1, p q},
\end{aligned}
$$

where $q_{l}^{1, \alpha \beta}=A_{l}^{\alpha} A_{l}^{\beta}=q_{l}^{2, \alpha \beta}+q_{l}^{3, \alpha \beta}$.

By (4.2), (4.3), (4.4) and similar evaluation, we have $\mathcal{B}_{n, h}^{1, p q}=\mathcal{B}_{n, h}^{2, p q}+O_{p}\left(b_{n}^{-1 / 2}\right)$, where $\mathcal{B}_{n, h}^{2, p q}$ is defined in the same way of $\mathcal{B}_{n, h}^{1, p q}$ with $q_{l}^{2, \alpha \beta}$ instead of $q_{l}^{1, \alpha \beta}$. It is easy to see that $\mathcal{T}_{n, h}^{1, p q}=O_{p}\left(h_{n}\right)$.

By simple calculus, we have

$$
\begin{aligned}
\mathcal{B}_{n, h}^{2, p q}= & \frac{9}{8} \sum_{l=2}^{b_{n}-n(h)} \sum_{k=1}^{n(h)-1}(n(h)-k) \\
& \times\left\{\left(q_{l}^{2, \alpha_{1} \alpha_{2}}\left(q_{l+k}^{2, \beta_{1} \beta_{2}}-q_{l}^{2, \beta_{1} \beta_{2}}\right)+q_{l}^{2, \alpha_{1} \beta_{2}}\left(q_{l+k}^{2, \beta_{1} \alpha_{2}}-q_{l}^{2, \beta_{1} \alpha_{2}}\right)\right.\right. \\
& \left.\left.\quad+\left(q_{l+k}^{2, \alpha_{1} \alpha_{2}}-q_{l}^{2, \alpha_{1} \alpha_{2}}\right) q_{l}^{2, \beta_{1} \beta_{2}}+\left(q_{l+k}^{2, \alpha_{1} \beta_{2}}-q_{l}^{2, \alpha_{1} \beta_{2}}\right) q_{l}^{2, \beta_{1} \alpha_{2}}\right)\right\} h_{n}^{-2}\left(\frac{T}{b_{n}}\right) \\
+ & \frac{9}{8} \sum_{l=2}^{b_{n}} n(h)^{2}\left(q_{l}^{2, \alpha_{1} \alpha_{2}} q_{l}^{2, \beta_{1} \beta_{2}}+q_{l}^{2, \alpha_{1} \beta_{2}} q_{l}^{2, \beta_{1} \alpha_{2}}\right) h_{n}^{-2}\left(\frac{T}{b_{n}}\right) \\
& -\frac{9}{8} \sum_{l=b_{n}-n(h)+1}^{b_{n}} n(h)^{2}\left(q_{l}^{2, \alpha_{1} \alpha_{2}} q_{l}^{2, \beta_{1} \beta_{2}}+q_{l}^{2, \alpha_{1} \beta_{2}} q_{l}^{2, \beta_{1} \alpha_{2}}\right) h_{n}^{-2}\left(\frac{T}{b_{n}}\right) \\
= & : \mathcal{P}_{n, h}^{2, p q}+\mathcal{C}_{n, h}^{2, p q}-\mathcal{T}_{n, h}^{2, p q} .
\end{aligned}
$$

From the proof of Lemma 3 in [26] and simple calculus, it is derived that $\mathcal{C}_{n, h}^{2, p q}=$ $\gamma^{p q}+o_{p}\left(b_{n}^{-1 / 2}\right)$. It is easy to see that $\mathcal{T}_{n, h}^{2, p q}=o_{p}\left(h_{n}\right)$. 
Furthermore, we have

$$
\begin{aligned}
& \mathcal{P}_{n, h}^{2, p q}=\frac{9}{8} \sum_{l=2}^{b_{n}-n(h)} \sum_{k=1}^{n(h)-1}(n(h)-k) \\
& \times\left\{\left(q_{l}^{2, \alpha_{1} \alpha_{2}} \sum_{m=1}^{k}\left(q_{l+m}^{2, \beta_{1} \beta_{2}}-q_{l+m-1}^{2, \beta_{1} \beta_{2}}\right)+q_{l}^{2, \alpha_{1} \beta_{2}} \sum_{m=1}^{k}\left(q_{l+m}^{2, \beta_{1} \alpha_{2}}-q_{l+m-1}^{2, \beta_{1} \alpha_{2}}\right)\right.\right. \\
& \left.\left.+\sum_{m=1}^{k}\left(q_{l+m}^{2, \alpha_{1} \alpha_{2}}-q_{l+m-1}^{2, \alpha_{1} \alpha_{2}}\right) q_{l}^{2, \beta_{1} \beta_{2}}+\sum_{m=1}^{k}\left(q_{l+m}^{2, \alpha_{1} \beta_{2}}-q_{l+m-1}^{2, \alpha_{1} \beta_{2}}\right) q_{l}^{2, \beta_{1} \alpha_{2}}\right)\right\} h_{n}^{-2}\left(\frac{T}{b_{n}}\right) \\
& =\frac{9}{8} \sum_{l=2}^{b_{n}-n(h)} \sum_{k=1}^{n(h)-1} \frac{(n(h)-k)(n(h)-k+1)}{2} \\
& \times\left\{\left(q_{l}^{2, \alpha_{1} \alpha_{2}}\left(q_{l+k}^{2, \beta_{1} \beta_{2}}-q_{l+k-1}^{2, \beta_{1} \beta_{2}}\right)+q_{l}^{2, \alpha_{1} \beta_{2}}\left(q_{l+k}^{2, \beta_{1} \alpha_{2}}-q_{l+k-1}^{2, \beta_{1} \alpha_{2}}\right)\right.\right. \\
& \left.\left.+\left(q_{l+k}^{2, \alpha_{1} \alpha_{2}}-q_{l+k-1}^{2, \alpha_{1} \alpha_{2}}\right) q_{l}^{2, \beta_{1} \beta_{2}}+\left(q_{l+k}^{2, \alpha_{1} \beta_{2}}-q_{l+k-1}^{2, \alpha_{1} \beta_{2}}\right) q_{l}^{2, \beta_{1} \alpha_{2}}\right)\right\} h_{n}^{-2}\left(\frac{T}{b_{n}}\right) \\
& =\frac{9}{8} \sum_{l=2}^{n(h)} \sum_{k=1}^{n(h)-l} \frac{(n(h)-k)(n(h)-k+1)}{2} \\
& \times\left\{\left(q_{l}^{2, \alpha_{1} \alpha_{2}}\left(q_{l+k}^{2, \beta_{1} \beta_{2}}-q_{l+k-1}^{2, \beta_{1} \beta_{2}}\right)+q_{l}^{2, \alpha_{1} \beta_{2}}\left(q_{l+k}^{2, \beta_{1} \alpha_{2}}-q_{l+k-1}^{2, \beta_{1} \alpha_{2}}\right)\right.\right. \\
& \left.\left.+\left(q_{l+k}^{2, \alpha_{1} \alpha_{2}}-q_{l+k-1}^{2, \alpha_{1} \alpha_{2}}\right) q_{l}^{2, \beta_{1} \beta_{2}}+\left(q_{l+k}^{2, \alpha_{1} \beta_{2}}-q_{l+k-1}^{2, \alpha_{1} \beta_{2}}\right) q_{l}^{2, \beta_{1} \alpha_{2}}\right)\right\} h_{n}^{-2}\left(\frac{T}{b_{n}}\right) \\
& +\frac{9}{8} \sum_{l^{\prime}=n(h)+1}^{b_{n}} \sum_{k=1}^{n(h)-1} \frac{(n(h)-k)(n(h)-k+1)}{2} \\
& \times\left\{\left(q_{l^{\prime}-k}^{2, \alpha_{1} \alpha_{2}}\left(q_{l^{\prime}}^{2, \beta_{1} \beta_{2}}-q_{l^{\prime}-1}^{2, \beta_{1} \beta_{2}}\right)+q_{l^{\prime}-k}^{2, \alpha_{1} \beta_{2}}\left(q_{l^{\prime}}^{2, \beta_{1} \alpha_{2}}-q_{l^{\prime}-1}^{2, \beta_{1} \alpha_{2}}\right)\right.\right. \\
& \left.\left.+\left(q_{l^{\prime}}^{2, \alpha_{1} \alpha_{2}}-q_{l^{\prime}-1}^{2, \alpha_{1} \alpha_{2}}\right) q_{l^{\prime}-k}^{2, \beta_{1} \beta_{2}}+\left(q_{l^{\prime}}^{2, \alpha_{1} \beta_{2}}-q_{l^{\prime}-1}^{2, \alpha_{1} \beta_{2}}\right) q_{l^{\prime}-k}^{2, \beta_{1} \alpha_{2}}\right)\right\} h_{n}^{-2}\left(\frac{T}{b_{n}}\right) \\
& -\frac{9}{8} \sum_{l=b_{n}-n(h)+1}^{b_{n}-1} \sum_{k=1}^{b_{n}-l} \frac{(n(h)-k)(n(h)-k+1)}{2} \\
& \times\left\{\left(q_{l}^{2, \alpha_{1} \alpha_{2}}\left(q_{l+k}^{2, \beta_{1} \beta_{2}}-q_{l+k-1}^{2, \beta_{1} \beta_{2}}\right)+q_{l}^{2, \alpha_{1} \beta_{2}}\left(q_{l+k}^{2, \beta_{1} \alpha_{2}}-q_{l+k-1}^{2, \beta_{1} \alpha_{2}}\right)\right.\right. \\
& \left.\left.+\left(q_{l+k}^{2, \alpha_{1} \alpha_{2}}-q_{l+k-1}^{2, \alpha_{1} \alpha_{2}}\right) q_{l}^{2, \beta_{1} \beta_{2}}+\left(q_{l+k}^{2, \alpha_{1} \beta_{2}}-q_{l+k-1}^{2, \alpha_{1} \beta_{2}}\right) q_{l}^{2, \beta_{1} \alpha_{2}}\right)\right\} h_{n}^{-2}\left(\frac{T}{b_{n}}\right) \\
& =: \mathcal{H}_{n, h}^{3, p q}+\mathcal{P}_{n, h}^{3, p q}-\mathcal{T}_{n, h}^{3, p q} \text {. } \\
& \mathcal{H}_{n, h}^{3, p q}=O_{p}\left(h_{n}\right) \text { and } \mathcal{T}_{n, h}^{3, p q}=O_{p}\left(h_{n}\right) \text { are derived from the following calculus. } \\
& \frac{(n(h)-k)(n(h)-k+1)}{2}\left(q_{l+k}^{2, \beta_{1} \beta_{2}}-q_{l+k-1}^{2, \beta_{1} \beta_{2}}\right) \\
& =\frac{(n(h)-k)(n(h)-(k-1))}{2} q_{l+k}^{2, \beta_{1} \beta_{2}}-\frac{(n(h)-(k-1))(n(h)-(k-2))}{2} q_{l+(k-1)}^{2, \beta_{1} \beta_{2}} \\
& +(n(h)-(k-1)) q_{l+(k-1)}^{2, \beta_{1} \beta_{2}} .
\end{aligned}
$$


Table 1: MSE of asymptotic variance estimators; $a_{n}=b_{n}^{2}$

\begin{tabular}{rrrrrrrr}
\hline$*$ & $b_{n}=2^{4}$ & $b_{n}=2^{5}$ & $b_{n}=2^{6}$ & $b_{n}=2^{7}$ & $b_{n}=2^{8}$ & $b_{n}=2^{9}$ & $b_{n}=2^{10}$ \\
\hline 1 & 0.6514 & 0.6775 & 0.6931 & 0.6835 & 0.6892 & 0.6923 & 0.6908 \\
2 & 0.4758 & 0.5838 & 0.6427 & 0.6537 & 0.6749 & 0.6845 & 0.6865 \\
$\mathrm{w}$ & 0.2102 & 0.2279 & 0.2783 & 0.3554 & 0.3980 & 0.4213 & 0.4667 \\
$\mathrm{~m}$ & 0.6063 & 0.3973 & 0.7440 & 0.6470 & 0.7390 & 0.6298 & 0.7188 \\
$\mathrm{n}$ & 1.3349 & 0.7355 & 0.3196 & 0.8940 & 1.3771 & 0.5679 & 0.6630 \\
\hline
\end{tabular}

Here, we set

$$
\mathcal{P}_{n, h}^{3, p q}=\sum_{l^{\prime}=n(h)+1}^{b_{n}} p_{n, h, l^{\prime}}^{3, p q}
$$

and we have

$$
\begin{aligned}
\sum_{l^{\prime}=n(h)+1}^{b_{n}} E\left[p_{n, h, l^{\prime}}^{3, p q} \mid \mathcal{F}_{l^{\prime}-3}^{n}\right] & =O_{p}\left(h_{n}\right), \\
\sum_{l^{\prime}=n(h)+1}^{b_{n}} E\left[\left|p_{n, h, l^{\prime}}^{3, p q}\right|^{2} \mid \mathcal{F}_{l^{\prime}-3}^{n}\right] & =O_{p}\left(h_{n}^{2}\right)
\end{aligned}
$$

and $\left\{p_{n, h, l^{\prime}}^{3, p q}\right\}_{l^{\prime}=2}^{b_{n}}$ is $\left\{\mathcal{F}_{l^{\prime}-2}^{n}\right\}_{l^{\prime}=2}^{b_{n}}$-adapted. Therefore, by Lenglart's inequality and its application, it holds that $\mathcal{P}_{n, h}^{3, p q}=O_{p}\left(h_{n}\right)$.

Here, we proved that $G_{n, h}^{p q}=\gamma^{p q}+o_{p}\left(b_{n}^{-1 / 2}\right)+O_{p}\left(b_{n}^{-1 / 2}\right)+O_{p}\left(h_{n}\right)$. We reached the conclusion.

\section{Simulation}

We consider the following example. $\mathbb{X}$ is an $\mathbb{R}_{+}^{2}$-valued Itô process satisfying

$$
\begin{aligned}
X_{t}^{1} & =X_{0}^{1}+\int_{0}^{t} X_{s}^{1} \mu_{1} d s+\int_{0}^{t} X_{s}^{1} \sigma_{1} d w_{s}^{1} \\
X_{t}^{2} & =X_{0}^{2}+\int_{0}^{t} X_{s}^{2} \mu_{2} d s+\int_{0}^{t} X_{s}^{2} \rho \sigma_{2} d w_{s}^{1}+\int_{0}^{t} X_{s}^{2} \sqrt{1-\rho^{2}} \sigma_{2} d w_{s}^{2}
\end{aligned}
$$

where $w^{1}$ and $w^{2}$ are independent Wiener processes, and $t \in[0, T]$. $\mathbb{Y}$ is a counting process with intensity process $a_{n} \mathbb{X}$. In this setting, $[\mathrm{C}]$ is satisfied.

In various $a_{n}, b_{n}$ settings, We examine the asymptotic variance estimators $\hat{\Xi}_{n, *}$, for $*=1,2, w, m, n$, where $\hat{\Xi}_{n, w}=\hat{\Xi}_{n, h}, h=h_{n}=T b_{n}^{-0.25} ; \hat{\Xi}_{n, m}=\hat{\Xi}_{n, h}$, $h=h_{n}=T b_{n}^{-0.5} ; \hat{\Xi}_{n, n}=\hat{\Xi}_{n, h}, h=h_{n}=T b_{n}^{-0.75}$. To reflect the condition that $\lim _{n \rightarrow \infty} b_{n}^{r} / a_{n}=0(r=2,2.5,3)$, we see the case that $a_{n}=b_{n}^{r}(r=2,2.5,3,3.5)$. We calculate these with 1000 paths and compare these by mean squared error (MSE).

Now, we fix the parameters $\mu_{1}=0.2, \mu_{2}=0.3, \sigma_{1}=0.2, \sigma_{2}=0.3, \rho=$ $0.7, X_{0}^{1}=1, X_{0}^{2}=2, T=1$.

Table 1, 2, 3 and 4 display the MSE of the asymptotic variance estimators in the case that $a_{n}=b_{n}^{r}(r=2,2.5,3,3.5)$, respectively. Table 1 and 2 show 
Table 2: MSE of asymptotic variance estimators; $a_{n}=b_{n}^{2.5}$

\begin{tabular}{rrrrrrrr}
\hline$*$ & $b_{n}=2^{4}$ & $b_{n}=2^{5}$ & $b_{n}=2^{6}$ & $b_{n}=2^{7}$ & $b_{n}=2^{8}$ & $b_{n}=2^{9}$ & $b_{n}=2^{10}$ \\
\hline 1 & 0.6634 & 0.6204 & 0.6516 & 0.6101 & 0.5332 & 0.4594 & 0.3712 \\
2 & 0.4831 & 0.5289 & 0.5987 & 0.5828 & 0.5212 & 0.4540 & 0.3690 \\
$\mathrm{w}$ & 0.2066 & 0.2113 & 0.2463 & 0.2918 & 0.2918 & 0.2617 & 0.2317 \\
$\mathrm{~m}$ & 0.5864 & 0.3543 & 0.6548 & 0.5325 & 0.5496 & 0.4013 & 0.3769 \\
$\mathrm{n}$ & 1.2788 & 0.6335 & 0.2680 & 0.7180 & 0.9890 & 0.3297 & 0.3145 \\
\hline
\end{tabular}

Table 3: MSE of asymptotic variance estimators; $a_{n}=b_{n}^{3}$

\begin{tabular}{rrrrrrrr}
\hline$*$ & $b_{n}=2^{4}$ & $b_{n}=2^{5}$ & $b_{n}=2^{6}$ & $b_{n}=2^{7}$ & $b_{n}=2^{8}$ & $b_{n}=2^{9}$ & $b_{n}=2^{10}$ \\
\hline 1 & 0.5676 & 0.4363 & 0.2485 & 0.1052 & 0.0364 & 0.0113 & 0.0038 \\
2 & 0.4185 & 0.3663 & 0.2259 & 0.0994 & 0.0351 & 0.0110 & 0.0037 \\
$\mathrm{w}$ & 0.1854 & 0.1289 & 0.0736 & 0.0344 & 0.0106 & 0.0025 & 0.0013 \\
$\mathrm{~m}$ & 0.5149 & 0.2247 & 0.2333 & 0.0798 & 0.0348 & 0.0069 & 0.0034 \\
$\mathrm{n}$ & 1.1188 & 0.4379 & 0.0818 & 0.1119 & 0.0885 & 0.0037 & 0.0018 \\
\hline
\end{tabular}

Table 4: MSE of asymptotic variance estimators; $a_{n}=b_{n}^{3.5}$

\begin{tabular}{rrrrrrrr}
\hline$*$ & $b_{n}=2^{4}$ & $b_{n}=2^{5}$ & $b_{n}=2^{6}$ & $b_{n}=2^{7}$ & $b_{n}=2^{8}$ & $b_{n}=2^{9}$ & $b_{n}=2^{10}$ \\
\hline 1 & 0.3284 & 0.1144 & 0.0423 & 0.0135 & 0.0058 & 0.0027 & 0.0015 \\
2 & 0.2468 & 0.0948 & 0.0391 & 0.0128 & 0.0057 & 0.0027 & 0.0015 \\
$\mathrm{w}$ & 0.1003 & 0.0288 & 0.0132 & 0.0082 & 0.0059 & 0.0043 & 0.0027 \\
$\mathrm{~m}$ & 0.2784 & 0.0471 & 0.0313 & 0.0086 & 0.0041 & 0.0017 & 0.0009 \\
$\mathrm{n}$ & 0.6123 & 0.1014 & 0.0142 & 0.0125 & 0.0147 & 0.0025 & 0.0010 \\
\hline
\end{tabular}

Table 5: $b_{n} \times$ MSE of asymptotic variance estimators; $a_{n}=b_{n}^{2}$

\begin{tabular}{rrrrrrrr}
\hline$*$ & $b_{n}=2^{4}$ & $b_{n}=2^{5}$ & $b_{n}=2^{6}$ & $b_{n}=2^{7}$ & $b_{n}=2^{8}$ & $b_{n}=2^{9}$ & $b_{n}=2^{10}$ \\
\hline 1 & 10.4229 & 21.6803 & 44.3604 & 87.4912 & 176.4313 & 354.4660 & 707.3375 \\
2 & 7.6130 & 18.6801 & 41.1353 & 83.6707 & 172.7787 & 350.4819 & 702.9933 \\
$\mathrm{w}$ & 3.3640 & 7.2915 & 17.8084 & 45.4855 & 101.8822 & 215.6920 & 477.9334 \\
$\mathrm{~m}$ & 9.7003 & 12.7150 & 47.6132 & 82.8206 & 189.1759 & 322.4385 & 736.0519 \\
$\mathrm{n}$ & 21.3576 & 23.5368 & 20.4572 & 114.4274 & 352.5406 & 290.7497 & 678.8655 \\
\hline
\end{tabular}

Table 6: $b_{n} \times$ MSE of asymptotic variance estimators; $a_{n}=b_{n}^{2.5}$

\begin{tabular}{rrrrrrrr}
\hline$*$ & $b_{n}=2^{4}$ & $b_{n}=2^{5}$ & $b_{n}=2^{6}$ & $b_{n}=2^{7}$ & $b_{n}=2^{8}$ & $b_{n}=2^{9}$ & $b_{n}=2^{10}$ \\
\hline 1 & 10.6144 & 19.8530 & 41.7007 & 78.0909 & 136.5013 & 235.2049 & 380.0606 \\
2 & 7.7301 & 16.9243 & 38.3189 & 74.5957 & 133.4145 & 232.4373 & 377.8717 \\
$\mathrm{w}$ & 3.3050 & 6.7621 & 15.7652 & 37.3484 & 74.6952 & 134.0158 & 237.2865 \\
$\mathrm{~m}$ & 9.3827 & 11.3368 & 41.9059 & 68.1650 & 140.6983 & 205.4797 & 385.9489 \\
$\mathrm{n}$ & 20.4606 & 20.2736 & 17.1543 & 91.9000 & 253.1752 & 168.8176 & 322.0486 \\
\hline
\end{tabular}


Table 7: $b_{n} \times$ MSE of asymptotic variance estimators; $a_{n}=b_{n}^{3}$

\begin{tabular}{rrrrrrrr}
\hline$*$ & $b_{n}=2^{4}$ & $b_{n}=2^{5}$ & $b_{n}=2^{6}$ & \multicolumn{1}{c}{$b_{n}=2^{7}$} & $b_{n}=2^{8}$ & $b_{n}=2^{9}$ & $b_{n}=2^{10}$ \\
\hline 1 & 9.0823 & 13.9603 & 15.9025 & 13.4609 & 9.3177 & 5.7789 & 3.8676 \\
2 & 6.6952 & 11.7201 & 14.4583 & 12.7193 & 8.9962 & 5.6229 & 3.7991 \\
$\mathrm{w}$ & 2.9661 & 4.1263 & 4.7102 & 4.4094 & 2.7073 & 1.2673 & 1.3712 \\
$\mathrm{~m}$ & 8.2390 & 7.1898 & 14.9288 & 10.2106 & 8.9112 & 3.5153 & 3.4378 \\
$\mathrm{n}$ & 17.9001 & 14.0135 & 5.2371 & 14.3203 & 22.6665 & 1.9175 & 1.7924 \\
\hline
\end{tabular}

Table 8: $b_{n} \times$ MSE of asymptotic variance estimators; $a_{n}=b_{n}^{3.5}$

\begin{tabular}{rrrrrrrr}
\hline$*$ & $b_{n}=2^{4}$ & $b_{n}=2^{5}$ & $b_{n}=2^{6}$ & $b_{n}=2^{7}$ & $b_{n}=2^{8}$ & $b_{n}=2^{9}$ & $b_{n}=2^{10}$ \\
\hline 1 & 5.2550 & 3.6621 & 2.7055 & 1.7321 & 1.4969 & 1.3790 & 1.4981 \\
2 & 3.9496 & 3.0341 & 2.4992 & 1.6330 & 1.4694 & 1.3785 & 1.4858 \\
$\mathrm{w}$ & 1.6055 & 0.9202 & 0.8457 & 1.0472 & 1.4993 & 2.1785 & 2.7900 \\
$\mathrm{~m}$ & 4.4538 & 1.5082 & 2.0064 & 1.0981 & 1.0475 & 0.8577 & 0.8826 \\
$\mathrm{n}$ & 9.7961 & 3.2457 & 0.9092 & 1.5997 & 3.7559 & 1.2657 & 1.0496 \\
\hline
\end{tabular}

that the MSE of the estimators do not converge to 0. Table 3 and 4 show that they converge to 0. These support Corollary 3.3 (a) and Corollary 3.4 (a).

Table 5, 6, 7 and 8 display $b_{n}$ times the MSE of the asymptotic variance estimators in the case that $a_{n}=b_{n}^{r}(r=2,2.5,3,3.5)$, respectively. Table 5] and 6] show that $b_{n}$ times the MSE of the estimators do not converge to 0. Table 7 and 8 show that $b_{n}$ times the MSE of $\hat{\Xi}_{n, *}(*=1,2, m, n)$ converge to 0 , and $b_{n}$ times the MSE of $\hat{\Xi}_{n, w}$ does not. These support Corollary 3.3 (c) and Corollary 3.4 (c).

\section{Acknowledgment}

The author would like to thank his supervisor Prof. Nakahiro Yoshida who provided valuable suggestions and comments.

\section{References}

[1] Abergel, F., Huré, C., Pham, H.: Algorithmic trading in a microstructural limit order book model. ArXiv e-prints (2017)

[2] Abergel, F., Jedidi, A.: A mathematical approach to order book modeling. International Journal of Theoretical and Applied Finance 16(05), 1350,025 $(2013)$

[3] Abergel, F., Jedidi, A.: Long-time behavior of a Hawkes process-based limit order book. SIAM Journal on Financial Mathematics 6(1), 1026$1043(2015)$

[4] Aït-Sahalia, Y., Fan, J., Xiu, D.: High-frequency covariance estimates with noisy and asynchronous financial data. Journal of the American Statistical Association 105(492), 1504-1517 (2010) 
[5] Bacry, E., Delattre, S., Hoffmann, M., Muzy, J.F.: Modelling microstructure noise with mutually exciting point processes. Quantitative Finance 13(1), 65-77 (2013)

[6] Barndorff-Nielsen, O.E., Hansen, P.R., Lunde, A., Shephard, N.: Multivariate realised kernels: consistent positive semi-definite estimators of the covariation of equity prices with noise and non-synchronous trading. Journal of Econometrics 162(2), 149-169 (2011)

[7] Barndorff-Nielsen, O.E., Shephard, N.: Econometric analysis of realized covariation: High frequency based covariance, regression, and correlation in financial economics. Econometrica 72(3), 885-925 (2004). URL http://www.jstor.org/stable/3598838

[8] Bibinger, M.: Efficient covariance estimation for asynchronous noisy highfrequency data. Scandinavian Journal of Statistics 38(1), 23-45 (2011)

[9] Bibinger, M.: An estimator for the quadratic covariation of asynchronously observed Itô processes with noise: asymptotic distribution theory. Stochastic Processes and their Applications 122(6), 2411-2453 (2012)

[10] Bowsher, C.G.: Modelling security market events in continuous time: Intensity based, multivariate point process models. Journal of Econometrics 141(2), 876-912 (2007)

[11] Chertok, A.V., Korolev, V.Y., Korchagin, A.Y.: Modeling high-frequency non-homogeneous order flows by compound Cox processes*. Journal of Mathematical Sciences 214(1), 44-68 (2016). DOI 10.1007/s10958-016-2757-6. URL https://doi.org/10.1007/s10958-016-2757-6

[12] Christensen, K., Kinnebrock, S., Podolskij, M.: Pre-averaging estimators of the ex-post covariance matrix in noisy diffusion models with nonsynchronous data. Journal of Econometrics 159(1), 116-133 (2010)

[13] Christensen, K., Podolskij, M., Vetter, M.: On covariation estimation for multivariate continuous Itô semimartingales with noise in non-synchronous observation schemes. Journal of Multivariate Analysis 120, 59-84 (2013)

[14] Clinet, S., Yoshida, N.: Statistical inference for ergodic point processes and application to limit order book. Stochastic Processes and their Applications 127(6), $1800 \quad$ - 1839 (2017). DOI http://dx.doi.org/10.1016/j.spa.2016.09.014. URL http://www.sciencedirect.com/science/article/pii/S0304414916301740

[15] Cont, R., Stoikov, S., Talreja, R.: A stochastic model for order book dynamics. Operations research 58(3), 549-563 (2010)

[16] Epps, T.W.: Comovements in stock prices in the very short run. Journal of the American Statistical Association 74(366a), 291-298 (1979)

[17] Glosten, L.R., Milgrom, P.R.: Bid, ask and transaction prices in a specialist market with heterogeneously informed traders. Journal of Financial Economics 14(1), $71 \quad-100$ (1985). 
DOI http://dx.doi.org/10.1016/0304-405X(85)90044-3.

URL http://www.sciencedirect.com/science/article/pii/0304405X85900443

[18] Gottlieb, G., Kalay, A.: Implications of the discreteness of observed stock prices. The Journal of Finance 40(1), 135-153 (1985). URL http://www.jstor.org/stable/2328052

[19] Griffin, J.E., Oomen, R.C.: Covariance measurement in the presence of non-synchronous trading and market microstructure noise. Journal of Econometrics 160(1), 58-68 (2011)

[20] Guilbaud, F., Pham, H.: Optimal High Frequency Trading with limit and market orders. ArXiv e-prints (2011)

[21] Hayashi, T., Yoshida, N.: Asymptotic normality of a covariance estimator for nonsynchronously observed diffusion processes. Annals of the Institute of Statistical Mathematics 60(2), 367-406 (2008)

[22] Hayashi, T., Yoshida, N.: Nonsynchronous covariation process and limit theorems. Stochastic Processes and their Applications 121(10), 2416-2454 (2011)

[23] Hayashi, T., Yoshida, N., et al.: On covariance estimation of nonsynchronously observed diffusion processes. Bernoulli 11(2), 359-379 (2005)

[24] Hewlett, P.: Clustering of order arrivals, price impact and trade path optimisation. In: Workshop on Financial Modeling with Jump processes, Ecole Polytechnique, pp. 6-8 (2006)

[25] Jacod, J., Li, Y., Mykland, P.A., Podolskij, M., Vetter, M.: Microstructure noise in the continuous case: the pre-averaging approach. Stochastic processes and their applications 119(7), 2249-2276 (2009)

[26] Kimura, A., Yoshida, N.: Estimation of Correlation Between Latent Processes, pp. 131-146. Springer International Publishing, Cham (2016). DOI 10.1007/978-3-319-45875-5\_6. URL https://doi.org/10.1007/978-3-319-45875-5_6

[27] Koike, Y.: Estimation of integrated covariances in the simultaneous presence of nonsynchronicity, microstructure noise and jumps. Econometric Theory pp. 1-79 (2013)

[28] Koike, Y.: An estimator for the cumulative co-volatility of asynchronously observed semimartingales with jumps. Scandinavian Journal of Statistics 41(2), 460-481 (2014)

[29] Koike, Y.: Limit theorems for the pre-averaged Hayashi-Yoshida estimator with random sampling. Stochastic Processes and their Applications 124(8), 2699-2753 (2014)

[30] Korolev, V.Y., Chertok, A.V., Korchagin, A.Y., Zeifman, A.I.: Modeling high-frequency order flow imbalance by functional limit theorems for twosided risk processes. ArXiv e-prints (2014) 
[31] Large, J.: Measuring the resiliency of an electronic limit order book. Journal of Financial Markets 10(1), 1-25 (2007)

[32] Malliavin, P., Mancino, M.E.: Fourier series method for measurement of multivariate volatilities. Finance and Stochastics 6(1), 49-61 (2002)

[33] Malliavin, P., Mancino, M.E., Recchioni, M.C.: A non-parametric calibration of the HJM geometry: an application of Itô calculus to financial statistics. Japanese Journal of Mathematics 2(1), 55-77 (2007). DOI 10.1007/s11537-007-0666-7. URL https://doi.org/10.1007/s11537-007-0666-7

[34] Malliavin, P., Mancino, M.E., et al.: A Fourier transform method for nonparametric estimation of multivariate volatility. The Annals of Statistics 37(4), 1983-2010 (2009)

[35] Mancino, M.E., Sanfelici, S.: Estimating covariance via Fourier method in the presence of asynchronous trading and microstructure noise. Journal of financial econometrics 9(2), 367-408 (2011)

[36] Muni Toke, I.: "Market making" behaviour in an order book model and its impact on the bid-ask spread. ArXiv e-prints (2010)

[37] Muni Toke, I.: Stationary distribution of the volume at the best quote in a Poisson order book model. ArXiv e-prints (2015)

[38] Muni Toke, I., Pomponio, F.: Modelling trades-through in a limited order book using Hawkes processes. Economics discussion paper (2011-32) (2011)

[39] Muni Toke, I., Yoshida, N.: Modelling intensities of order flows in a limit order book. ArXiv e-prints (2016)

[40] Mykland, P.A.: A Gaussian calculus for inference from high frequency data. Annals of finance 8(2-3), 235-258 (2012)

[41] Ogihara, T., Yoshida, N.: Quasi likelihood analysis for point process regression models. preprint (2015)

[42] Park, S., Linton, O.B., et al.: Estimating the quadratic covariation matrix for an asynchronously observed continuous time signal masked by additive noise (2012)

[43] Podolskij, M., Vetter, M., Sommer, M.: Estimation of volatility functionals in the simultaneous presence of microstructure noise and jumps. Available at SSRN 950344 (2007)

[44] Roll, R.: A simple implicit measure of the effective bid-ask spread in an efficient market. The Journal of Finance 39(4), 1127-1139 (1984). URL http://www.jstor.org/stable/2327617

[45] Smith, E., Farmer, J.D., Gillemot, L.s., Krishnamurthy, S., et al.: Statistical theory of the continuous double auction. Quantitative finance $\mathbf{3}(6)$, 481-514 (2003) 
[46] Toke, I.M.: The order book as a queueing system: average depth and influence of the size of limit orders. Quantitative Finance 15(5), 795-808 (2015). DOI 10.1080/14697688.2014.963654. URL http://dx.doi.org/10.1080/14697688.2014.963654

[47] Voev, V., Lunde, A.: Integrated covariance estimation using high-frequency data in the presence of noise. Journal of Financial Econometrics 5(1), 68 $104(2007)$

[48] Zhang, L., Mykland, P.A., Aït-Sahalia, Y.: A tale of two time scales. Journal of the American Statistical Association (2012)

[49] Zhang, L., et al.: Efficient estimation of stochastic volatility using noisy observations: A multi-scale approach. Bernoulli 12(6), 1019-1043 (2006)

[50] Zhou, B.: High-frequency data and volatility in foreign-exchange rates. Journal of Business \& Economic Statistics 14(1), 45-52 (1996) 